\title{
Accelerating Digitalization - Ansatzpunkte zur Beschleunigung der Digitalisierung
}

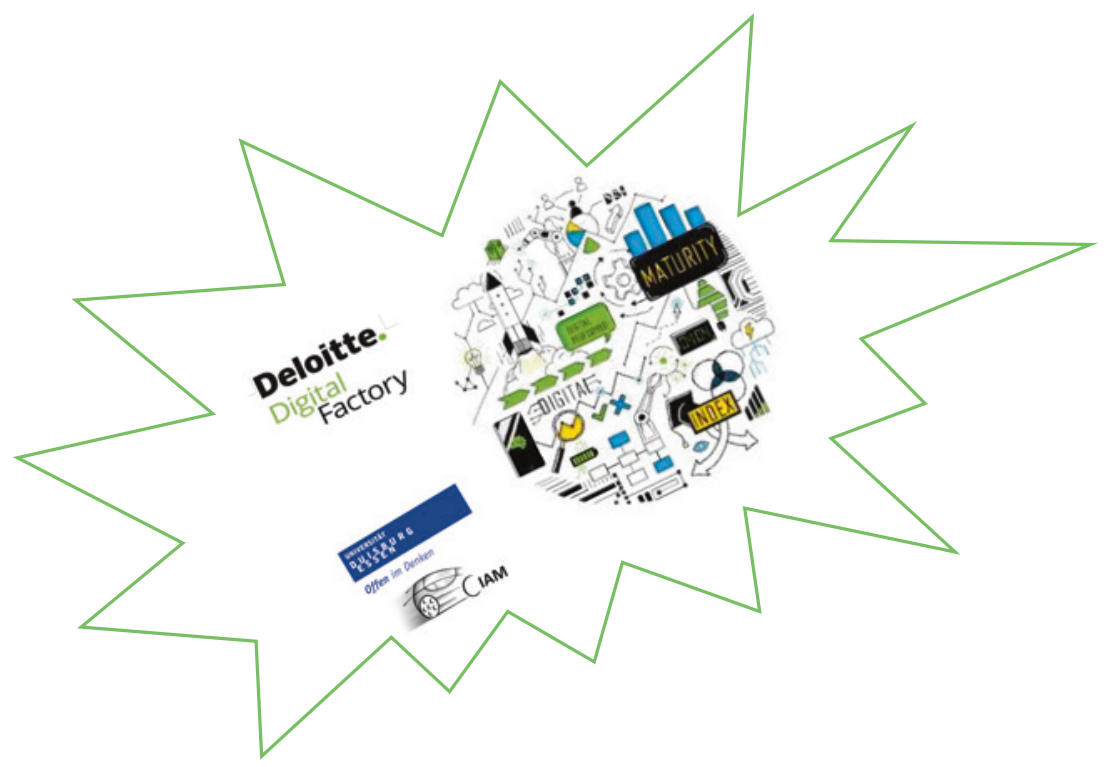

Elektronisches Zusatzmaterial Die elektronische Version dieses Kapitels enthält Zusatzmaterial, das berechtigten Benutzern zur Verfügung steht. https://doi. org/10.1007/978-3-658-31456-9_6. Die Videos lassen sich mit Hilfe der SN More Media App abspielen, wenn Sie die gekennzeichneten Abbildungen mit der App scannen. 
Wir entwickeln in diesem Kapitel Ansatzpunkte zur Beschleunigung der digitalen Transformation:

- Zunächst werden Wege aufgezeigt, wie die sechs in Kap. 5 unterschiedenen Archetypen digitaler Unter-nehmen die digitale Reife erhöhen können.

Dabei gilt es, konsequent die Schwachstellen zu beseitigen, die die vier Teilindizes (Digital Activity Index, Digtal Business Index, Dynamic Capability Index und Operational Capability Index) aufdecken.

- Anschließend wird die Notwendigkeit begründet, die Digitalisierung angesichts des zunehmenden Wettbewerbs um optimale digitale Leistungen weiter zu beschleunigen.

- Dafür werden Ansatzpunkte aufgezeigt:

- Vernetzung und Skalierung von Leistungen über geeignete digitale Schlüssel - Accelerator of Digitalization (1),

- rechtzeitige Quantifizierung von Einspar- und Erlöspotenzialen der Digitalisierung - Accelerator of Digitalization (2),

- Umsetzung digitaler Fähigkeiten durch minimal lebensfähige Leistungen, d.h. durch organisationale Agilität, die in kapitalintensiven Branchen die Notwendigkeit der Fixkostendegression durch Erzielung von Größen- und Verbundvorteilen berücksichtigen muss (hybride Agilität - Accelerator of Digitalization (3) und

- Schutz vor ungewolltem Datenabfluss (Cyber Security - Accelerator of Digitalization (4).

Im Kap. 5 wurde gezeigt, dass die meisten deutschen Unternehmen die Chancen der Digitalisierung (Kap.2) bisher noch nicht ausreichend nutzen - weder durch Digitalisierung ihrer Leistungen (Technologien, Prozesse, vor allem aber Angebote und Geschäftsmodelle, vgl. zu diesen Möglichkeiten Kap. 3), noch durch Aktivierung ihrer Veränderungsfähigkeiten und ihrer operativen Fähigkeiten (vgl. dazu Kap. 4). Damit erreichen sie bisher noch nicht die mögliche Gewinnwirkung.

Da sich Unternehmen hinsichtlich des Ausmaßes ihrer digitalen Reife unterscheiden, wurden sie sechs Archetypen zugeordnet (vgl. Abschn. 5.3). Für diese Archetypen werden in diesem Kapitel zunächst Wege zur Beschleunigung der digitalen Transformation gesucht (Abschn. 6.1). Dabei gibt es keine „Weltmeisterstrategie“ und nicht alle Unternehmen können sofort digitale Champions 
werden. Die Automobilindustrie muss z. B. mit dem Übergang in die Elektromobilität noch andere Baustellen bewältigen.

Um im weltweiten Wettbewerb um die Chancen der Digitalisierung ${ }^{1}$ nicht ins Hintertreffen zu geraten und um die durch die hohe Unsicherheit über die weitere Entwicklung digitaler Lösungen oft verzögerten Entscheidungen zu beschleunigen ${ }^{2}$, muss allerdings die Geschwindigkeit der digitalen Transformation weiter zunehmen (Abschn. 6.2). Zur Beschleunigung der digitalen Transformation werden vier Ansatzpunkte vorgeschlagen (Accelerators of Digitalization):

1. Leistungen über geeignete digitale Schlüssel vernetzen und skalieren (Abschn. 6.3),

2. Einspar- und Erlöspotenziale der Digitalisierung rechtzeitig quantifizieren (Abschn. 6.4),

3. digitale Fähigkeiten durch hybride Agilität in der Organisation umsetzen (Abschn. 6.5) und

4. Schutz vor ungewolltem Datenabfluss (Cyber Security) erhöhen (Abschn. 6.6).

\subsection{Wege zur Erhöhung der digitalen Reife}

Ausgangspunkt für die Erhöhung der digitalen Reife ist der Status quo, d. h. die in Kap. 5 bestimmte digitale Reife (Digital Maturity). Da es keine Weltmeisterstrategie für die weitere digitale Transformation gibt und im Rahmen dieses Buches auch keine individuellen Wege für einzelne Unternehmen aufgezeigt werden können, sollen Wege für die einzelnen Archetypen digitaler Unternehmen abgeleitet werden, um die Digitalisierungspotenziale noch besser nutzen zu können. Dabei werden allerdings nur die Archetypen 2 (digitale Potenzialträger) bis 6 (digitale Nachzügler) betrachtet, weil Unternehmen des Archetypen 1 (digitale Champions) die Chancen der Digitalisierung schon sehr weitgehend nutzen.

Die Archetypen wurden in Abschn. 5.3 mithilfe einer Clusteranalyse entlang der Diskriminanzachsen

- strategische Ausrichtung auf die Digitalisierung (Digital Strategy) und

- operative Ausrichtung auf die Digitalisierung (Digital Operations)

unterschieden (vgl. Abb. 5.10): vier Archetypen entlang der 45-Grad-Linie einer gleichmäßigen strategischen und operativen Digitalisierung und jeweils ein Archetyp ober- bzw. unterhalb dieser Linie. Dies entspricht dem Ergebnis in Abschn. 5.2, dass die strategische und die operative Ausrichtung auf die 
Digitalisierung in den befragten 160 Unternehmen mit einem $\mathrm{R}^{2}$ von 83 Prozent hoch korrelieren (vgl. Abb. 5.9a) und dass die Unternehmen deshalb ihre digitale Strategie und ihre Digital Operations gleichermaßen entwickeln.

Ansatzpunkte für die nun skizzierten Wege zur Erhöhung der digitalen Reife basieren für jeden der fünf Archetypen auf den Abweichungen bei den einzelnen Items der vier Teilindizes des Digital Maturity Index von den Archetypen mit einer höheren digitalen Reife. Für digitale Potenzialträger sind das also die Abweichungen von den digitalen Champions, für digitale Innovatoren die Abweichungen von den digitalen Potenzialträgern und ggf. auch von den digitalen Champions usw. Die Abweichungen werden als Durchschnitt für die einzelnen Archetypen digitaler Unternehmen berechnet.

\section{(1) Mögliche Wege der digitalen Nachzügler zur Erhöhung der digitalen Reife}

Digitale Nachzügler (Archetyp 6), die die digitale Transformation bislang noch am wenigsten angegangen haben, müssen die Chancen der Digitalisierung auf allen Dimensionen (bei Technologien und Prozessen, Angeboten und Geschäftsmodellen sowie Veränderungs- und operativen Fähigkeiten) besser nutzen. Auch wenn sie zumindest mit einzelnen Pilottechnologien und -prozessen schon begonnen haben, ist ihre digitale Reife erst so gering, dass sie einen Sprung bzw. ein „Leapfrogging ${ }^{\text {“3 }}$ zum digitalen Champion (Archetyp 1) nicht werden schaffen können. Wettbewerberreaktionen, technologische Veränderungen sowie der zunehmende Kompetenzrückstand im Zeitablauf dürften dafür zu groß sein. ${ }^{4}$

Den digitalen Nachzüglern bleibt nur

- eine schrittweise Verbesserung der Activities (Technologien und Prozesse) und Businesses (Angebote und Geschäftsmodelle) sowie der Dynamic und Operational Capabilities hin zu den digitalen Folgern, d. h. zu Archetyp 5 (Weg N.1).

Bei stärkerer digitaler Transformation werden sie

- entweder die strategische Ausrichtung durch verstärkte Digitalisierung der Angebote und Geschäftsmodelle sowie der Dynamic Capabilities hin zu den digitalen Innovatoren (Archetyp 3) verbessern (Weg N.2)

- oder die operative Ausrichtung durch verstärkte Digitalisierung der Prozesse sowie der operativen Fähigkeiten hin zu den digitalen Optimierern (Archetyp 4) noch weiter verbessern (Weg N.3). 
- Gelingt es ihnen, die strategische und operative Ausrichtung gleichermaßen sehr stark zu verbessern, können sie sogar zu einem digitalen Potenzialträger, d. h. Archetyp 2 aufschließen (Weg N.4).

Die vier möglichen Wege für die digitalen Nachzügler zu einer stärkeren digitalen Reife zeigt Abb. 6.1.

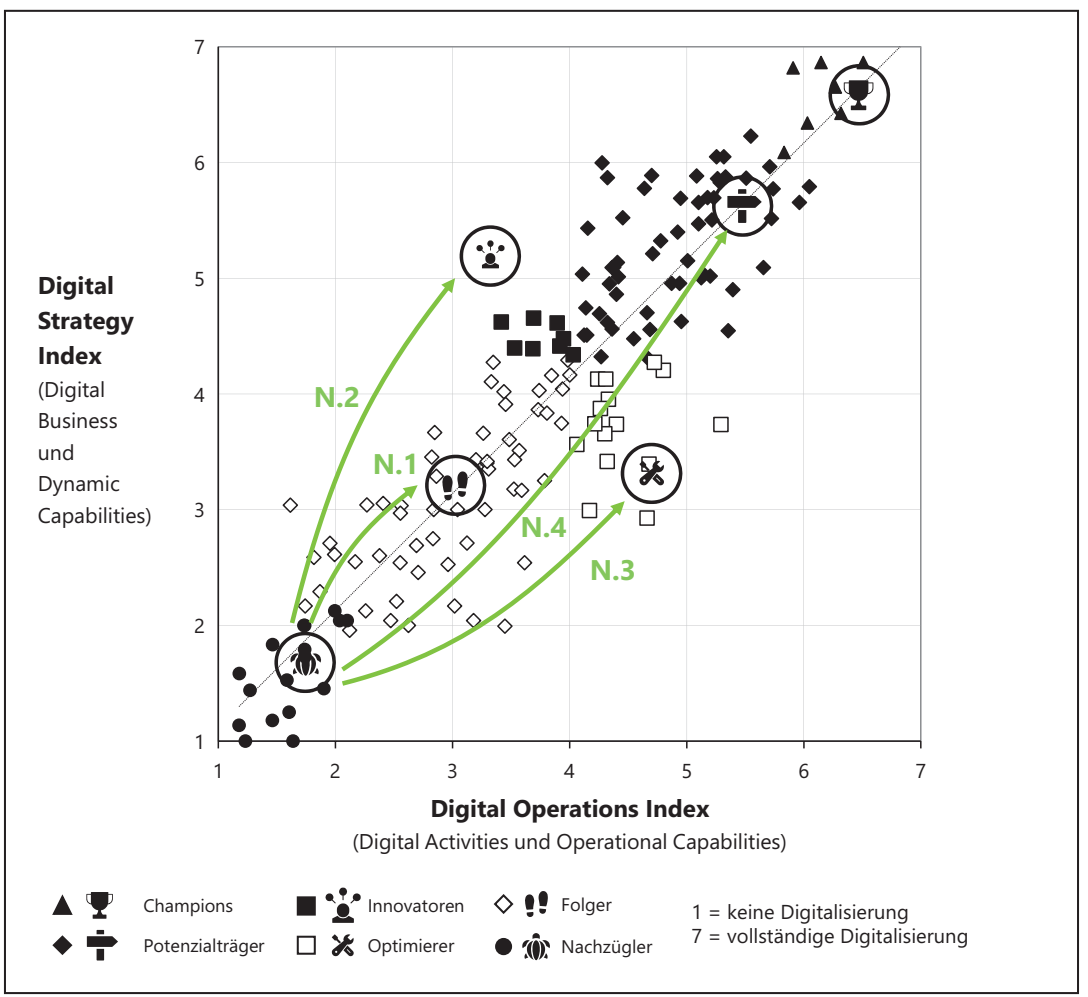

Abb. 6.1 Wege (N.1 bis N.4) der digitalen Nachzügler zur Erhöhung der digitalen Reife. (Quelle: eigener Entwurf) 
Der erste Weg der digitalen Nachzügler zu den digitalen Folgern (N.1) ergibt sich wie erwähnt aus den Abweichungen bei den einzelnen Items der vier Teilindizes. Die Ausgangswerte der Nachzügler entsprechen denen in Kap. 5, Abb. 5.16 und sind in Abb. 6.2 ebenfalls mit Radarcharts als Ränder der schwarzen Flächen dargestellt. Die Zielwerte der digitalen Folger liegen auf dem äußeren Datenkreis. Abb. 6.2 hebt die fünf stärksten Abweichungen zwischen beiden Archetypen besonders hervor (grün gedruckt). Bei ihnen sollte eine Verbesserung der digitalen Reife in besonderem Maße ansetzen, ohne darüber die anderen Items zu vergessen.

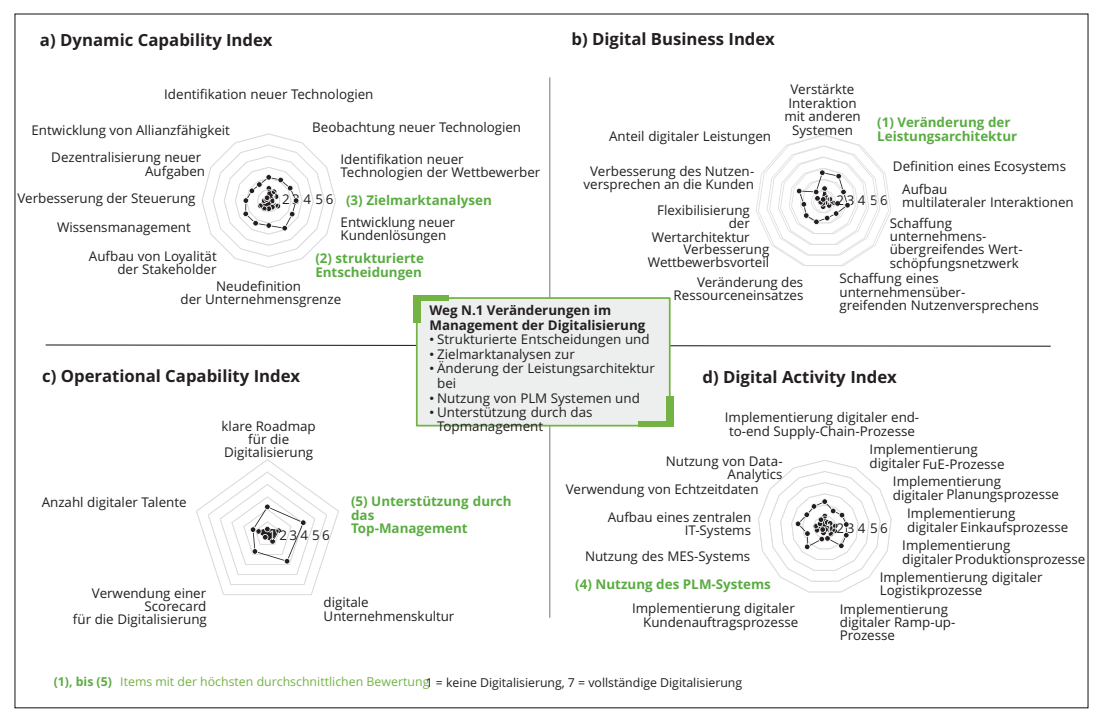

Abb. 6.2 Weg (N.1) der digitalen Nachzügler zu den digitalen Folgern. (Quelle: eigener Entwurf) 
Gemäß Abb. 6.2 müssen digitale Nachzügler auf dem Weg zum Follower vor allem

- Zielmarktanalysen und

- strukturierte Entscheidungen

einführen. Noch wichtiger als diese Veränderungsfähigkeiten sind jedoch

- eine Veränderung der Leistungsarchitektur der Angebote,

- die Technologie des Product Lifecycle Managements (PLM) zur Integration aller Informationen über den Lebenszyklus eines Produktes und

- eine Unterstützung durch das Top-Management.

Diese Ergebnisse entsprechen der Einschätzung, dass Digitalisierung weniger mit Technologie (PLM-Systeme) als mehr mit Strategien und Leistungen (Zielmarktanalysen und strukturieren Entscheidungen zur Veränderung der Angebote) zu tun hat. ${ }^{5}$ Gerade für Unternehmen, die noch am Anfang der digitalen Transformation stehen, ist diese Erkenntnis wichtig: Der Weg erfordert bessere Managementprozesse und Klarheit über die Angebote und Märkte. Der isolierte Einsatz weiterer digitaler Technologien hilft nicht weiter.

Wollen Unternehmen, die aufgrund ihrer bislang vernachlässigten digitalen Aktivitäten zu den digitalen Nachzüglern gehören, einen größeren Sprung in die Digitalisierung versuchen als nur an die digitalen Folger heranzureichen, können sie

- entweder eine noch stärkere Verbesserung ihrer strategischen Ausrichtung versuchen, um an die digitalen Innovatoren anzuschließen (Weg N.2),

- oder sich deutlich stärker operativ ausrichten, um die digitalen Optimierer zu erreichen (Weg N.3).

Abb. 6.3 zeigt im Vergleich mit Abb. 6.2 zunächst, dass der Sprung der digitalen Nachzügler zu den Innovatoren (Weg N.2) deutlich größer ist als der zu den Folgern auf Weg N.1 (die Zielwerte der Innovatoren auf dem äußeren Datenkreis in Abb. 6.3 sind deutlich weiter von den Rändern der schwarzen Ausgangsfläche der Nachzügler entfernt als die der Folger in Abb. 6.2). Um zu den Innovatoren aufzuschließen, müssen Nachzügler die digitale Transformation sehr stark auf strategische Maßnahmen konzentrieren.

Die fünf stärksten Abweichungen zwischen beiden Archetypen (grün gedruckt in Abb. 6.3), bei denen eine Verbesserung der digitalen Reife in besonderem Maße ansetzen sollte, betreffen entsprechend auch alle die strategische Ausrichtung auf die Digitalisierung durch 
- Zielmarktanalysen und

- strukturierte Entscheidungen sowie

- eine Veränderung der Leistungsarchitektur der Angebote.

Sie zielen zudem auch auf die Veränderung von Geschäftsmodellen durch.

- Änderung des Ressourceneinsatzes und

- Verbesserung der Wettbewerbsvorteile.

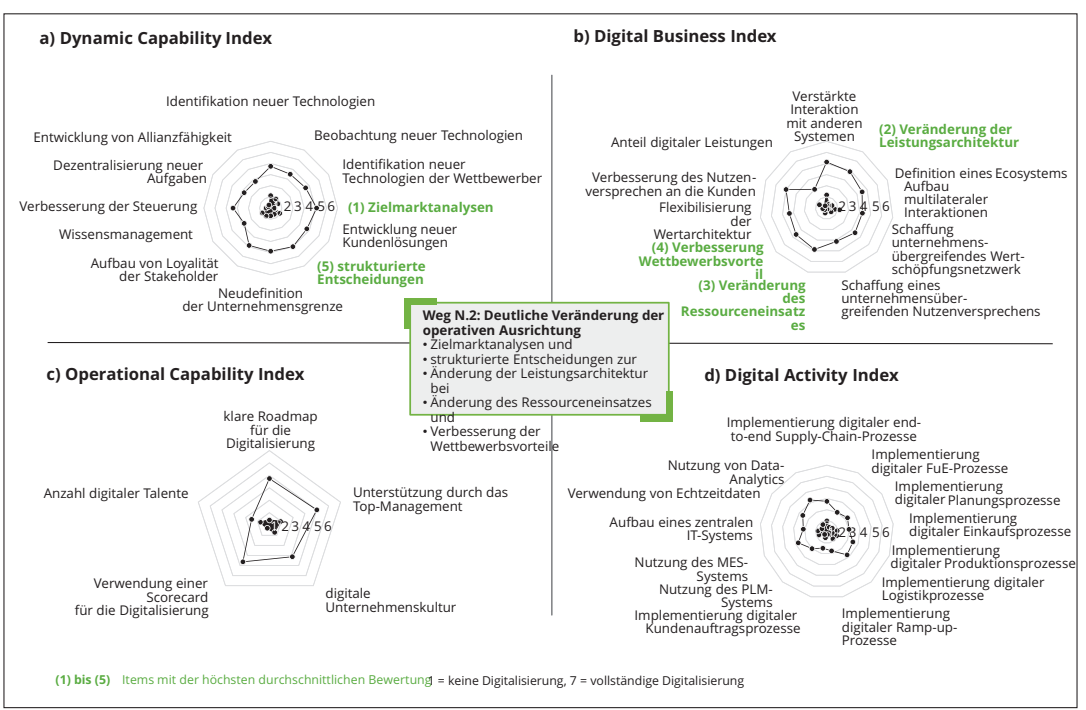

Abb.6.3 Weg (N.2) der digitalen Nachzügler zu den digitalen Innovatoren. (Quelle: eigener Entwurf)

Der Weg der digitalen Nachzügler zu den digitalen Optimierern (N.3) ist ebenfalls deutlich weiter als der zu den digitalen Folgern (N.1). Um zu den digitalen Optimierern aufzuschließen, müssen die Nachzügler die digitale Transformation ebenfalls nicht nur stärker beschleunigen, sondern sich - anders als im Übergang zu den digitalen Innovatoren in Abb. 6.3 - sehr stark auf operative Maßnahmen konzentrieren: Die fünf stärksten Abweichungen (grün gedruckt in Abb. 6.4) zwischen den Archetypen des Nachzüglers und des Optimierers, bei denen eine Verbesserung der digitalen Reife in besonderem Maße ansetzen sollte, betreffen entsprechend stark auch die operative Ausrichtung, d. h. einerseits operative Fähigkeiten wie 
- die Entwicklung einer digitalen Unternehmenskultur und

- die Verwendung einer Scorecard für die Digitalisierung und andererseits digitale Technologien und Prozesse wie

- die Nutzung von Echtzeitdaten,

- den Aufbau eines zentralen IT-Systems und

- die Implementierung digitaler Logistikprozesse.

Diese eher technologischen und prozessbezogenen Aktivitäten helfen, eine solide Basis für die Digitalisierung zu legen, werden aber den Gewinn allein noch nicht sehr stark verbessern, weil eine ergänzende Digitalisierung der Angebote und Geschäftsmodelle fehlt. Der Sprung zum digitalen Optimierer kann dennoch sinnvoll sein, um eine Basis für eine anschließend stärker strategische Ausrichtung auf die Digitalisierung zu schaffen. Digitale Nachzügler sollten bei diesem Weg allerdings nicht unterschätzen, wie schwer es ist, eine digitale Unternehmenskultur zu entwickeln.

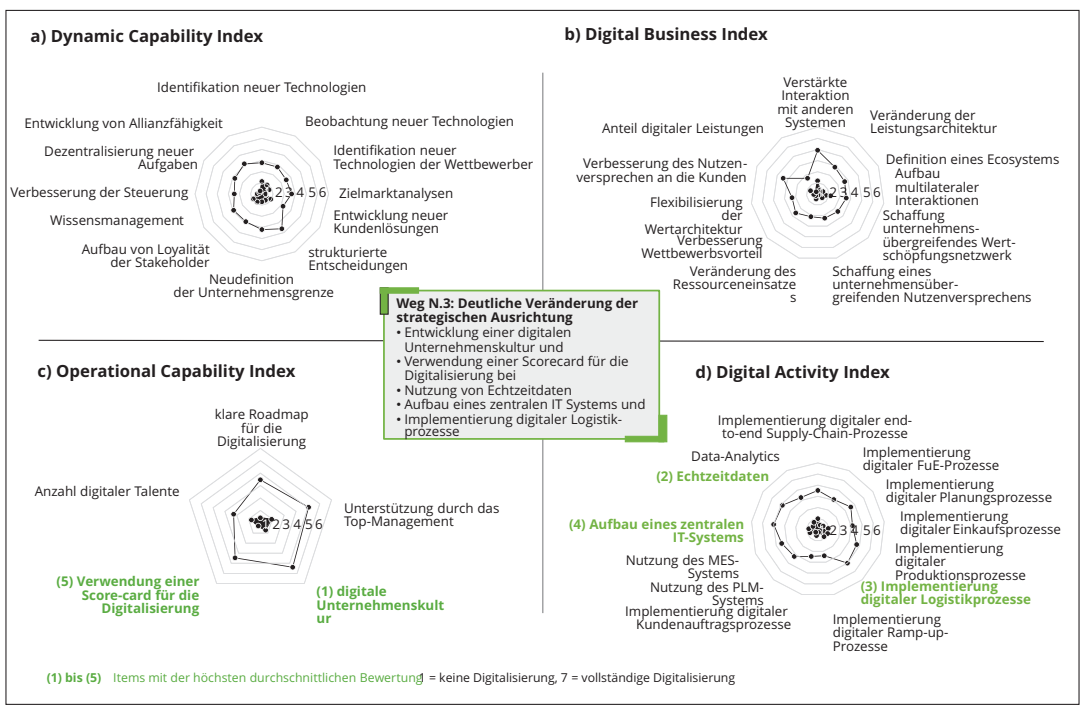

Abb.6.4 Weg (N.3) der digitalen Nachzügler zu den digitalen Optimierern. (Quelle: eigener Entwurf) 
Wollen Unternehmen, die zu den digitalen Nachzüglern gehören, die digitale Transformation noch stärker vorantreiben, können sie auch direkt den Weg vom Nachzügler zu den digitalen Potenzialträgern angehen (Weg N.4 in Abb. 6.5). Es sollte ihnen aber bewusst sein, dass dieser Sprung nochmals deutlich größer ist als der zu den digitalen Innovatoren oder Optimierern, weil sie ihre strategische und operative Ausrichtung auf die Digitalisierung gleichzeitig sehr stark verbessern müssen. Dies zeigen die großen Abweichungen zwischen den Rändern der schwarzen Ausgangsfläche als dem Status quo der digitalen Nachzügler und dem äußeren Datenkreis als den Zielwerten der digitalen Potenzialträger in Abb. 6.5. Weil dieser Sprung der schwierigste Weg für die digitalen Nachzügler ist, betreffen die fünf stärksten Abweichungen zwischen beiden Archetypen (grün gedruckt in Abb. 6.5), bei denen eine umfassende Verbesserung der digitalen Reife in besonderem Maße ansetzen sollte, auch alle Veränderungsfähigkeiten der Unternehmen.

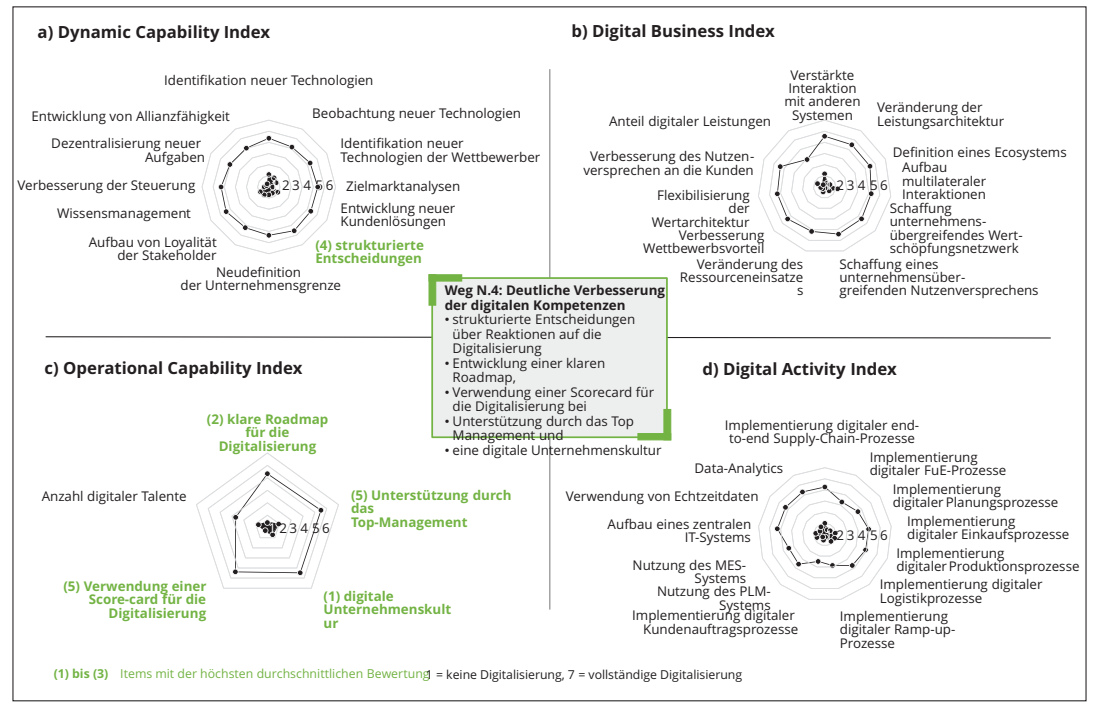

Abb. 6.5 Weg (N.4) der digitalen Nachzügler zu den digitalen Potenzialträgern. (Quelle: eigener Entwurf) 
Die fünf stärksten Abweichungen zwischen den Nachzüglern und den Potenzialträgern betreffen:

- strukturierte Entscheidungen über geeignete Reaktionen auf die Chancen der Digitalisierung, ergänzt durch umfassende operative Fähigkeiten zur

- Entwicklung einer klaren Roadmap, die

- durch Verwendung einer Scorecard für die Digitalisierung engmaschig nachgehalten wird sowie

- durch das Top-Management und

- eine digitale Unternehmenskultur unterstützt wird.

Für diesen schwierigen Weg müssen also erst die genannten Fähigkeiten aufgebaut werden, bevor die strategischen und operativen Leistungen in großem Umfang digitalisiert werden können.

\section{(2) Mögliche Wege der digitalen Folger zur Erhöhung der digitalen Reife}

Digitale Folger (Archetyp 5) haben die digitale Transformation bislang ebenfalls noch nicht sehr stark vorangetrieben und müssen die Chancen einer Weiterentwicklung auf allen Dimensionen (bei Technologien und Prozessen, Angeboten und Geschäftsmodellen sowie Veränderungsfähigkeiten und operativen Fähigkeiten) besser nutzen. Ähnlich, nur etwas weniger intensiv als die digitalen Nachzügler, können die digitalen Folger

- entweder die strategische Ausrichtung durch verstärkte Aktivierung der Veränderungsfähigkeiten und Digitalisierung der Angebote und Geschäftsmodelle hin zu den digitalen Innovatoren (Archetyp 3) verbessern (Weg F.1)

- oder die operative Ausrichtung durch verstärkte Digitalisierung der operativen Fähigkeiten und Digitalisierung der Technologien und Prozesse hin zu digitalen Optimierern (Archetyp 4) verbessern (Weg F.2).

- Die digitalen Folger können aber auch direkt die strategische und operative Ausrichtung gleichermaßen verbessern und dadurch einen Sprung zu den digitalen Potenzialträgern (Archetyp 2) versuchen (Weg F.3) (Abb. 6.6). 


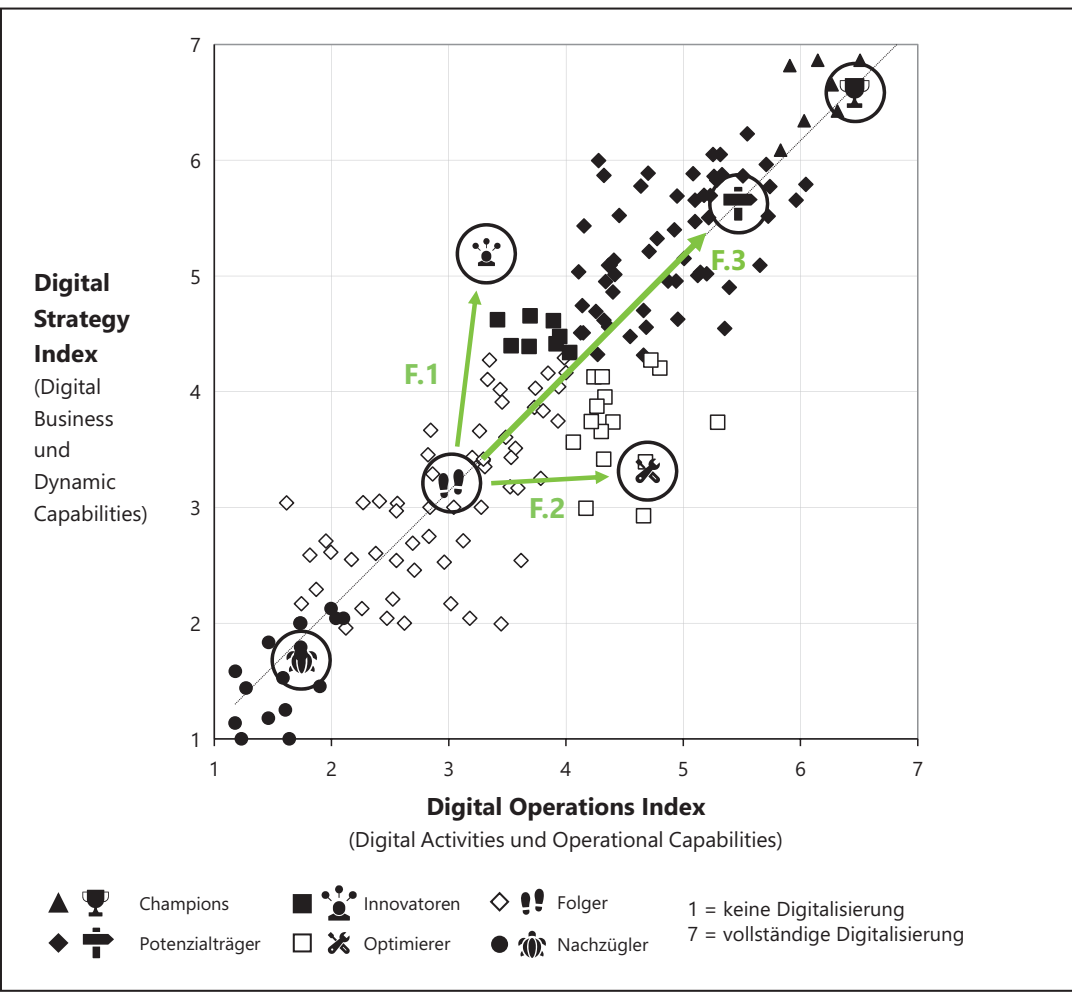

Abb. 6.6 Wege (F.1 bis F.3) der digitalen Folger zur Erhöhung der digitalen Reife. (Quelle: eigener Entwurf)

Für den ersten Weg der digitalen Folger zu den digitalen Innovatoren (Weg F.1) müssen sie sich auf strategische Maßnahmen zur Nutzung der Chancen der Digitalisierung konzentrieren. Es sind nämlich nicht nur die Abweichungen zwischen beiden Archetypen (vgl. Abb. 6.7) bei der strategischen Ausrichtung, d. h. bei den Veränderungsfähigkeiten (Dynamic Capabilities) sowie den Angeboten und Geschäftsmodellen (Businesses), am größten. Von den fünf stärksten Abweichungen (grün gedruckt in Abb. 6.7), bei denen eine Verbesserung der digitalen Reife in besonderem Maße ansetzen sollte, betreffen zudem vier die strategische Ausrichtung auf die Digitalisierung, v. a. die Geschäftsmodelle: 
- Veränderung des Ressourceneinsatzes,

- Verbesserung des Nutzenversprechens an die Kunden,

- Verbesserung des Wettbewerbsvorteils, aber auch die Digitalisierung der Angebote durch

- Ausrichtung auf ein unternehmensübergreifendes Wertschöpfungsnetzwerk bei gleichzeitiger Aktivierung von operativen Fähigkeiten der Ergebniskontrolle durch

- Verwendung einer Scorecard für die Digitalisierung.

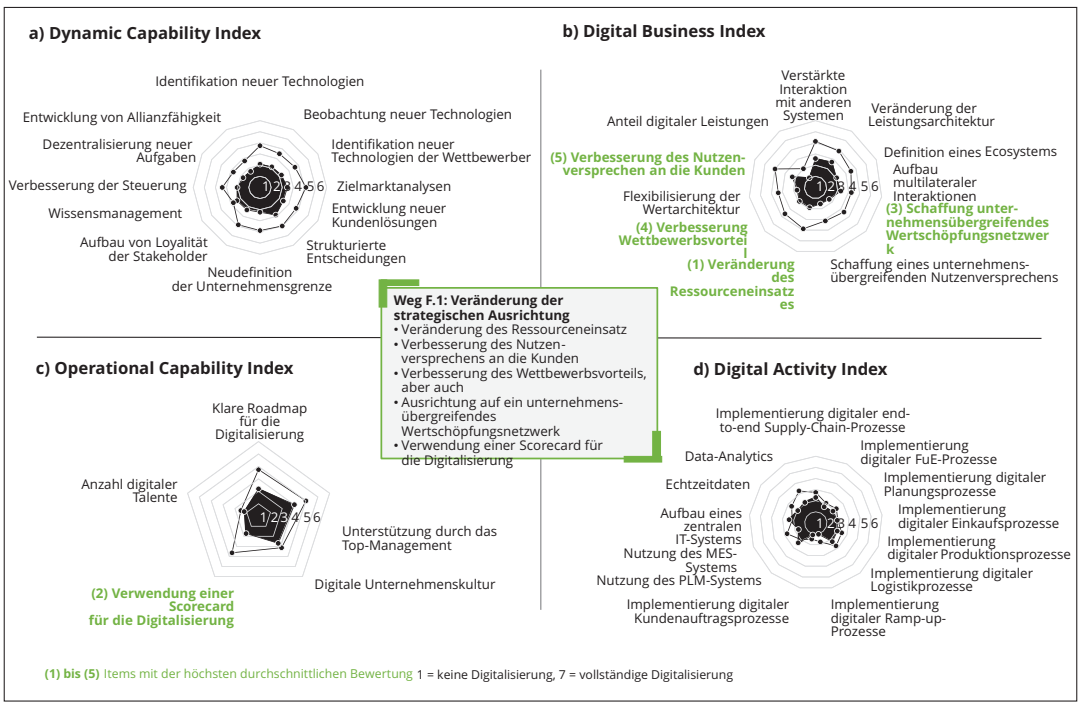

Abb. 6.7 Weg (F.1) der digitalen Folger zu den digitalen Innovatoren. (Quelle: Eigener Entwurf) 
Für den Weg der digitalen Folger zu den digitalen Optimierern (F.2) sind dagegen operative Maßnahmen erforderlich: Anders als im Übergang zu den Innovatoren sind nicht nur die Abweichungen zwischen beiden Archetypen (vgl. Abb. 6.8) bei der operativen Ausrichtung, d. h. bei den operativen Fähigkeiten, den Technologien und Prozessen (Activities), am größten. Von den fünf stärksten Abweichungen (grün gedruckt in Abb. 6.8), bei denen eine Verbesserung der digitalen Reife in besonderem Maße ansetzen sollte, betreffen zudem vier die operative Ausrichtung auf die Digitalisierung Sie betreffen insbesondere die Verbesserung der operativen Kontrollfähigkeit

- durch Verwendung einer Scorecard für die Digitalisierung, die Nutzung digitaler Technologien, v. a.

- die Nutzung von Echtzeitdaten und

- das Manufacturing Execution System (MES) sowie die Digitalisierung von Prozessen, insbesondere

- die Implementierung digitaler Logistik- und

- Planungsprozesse.

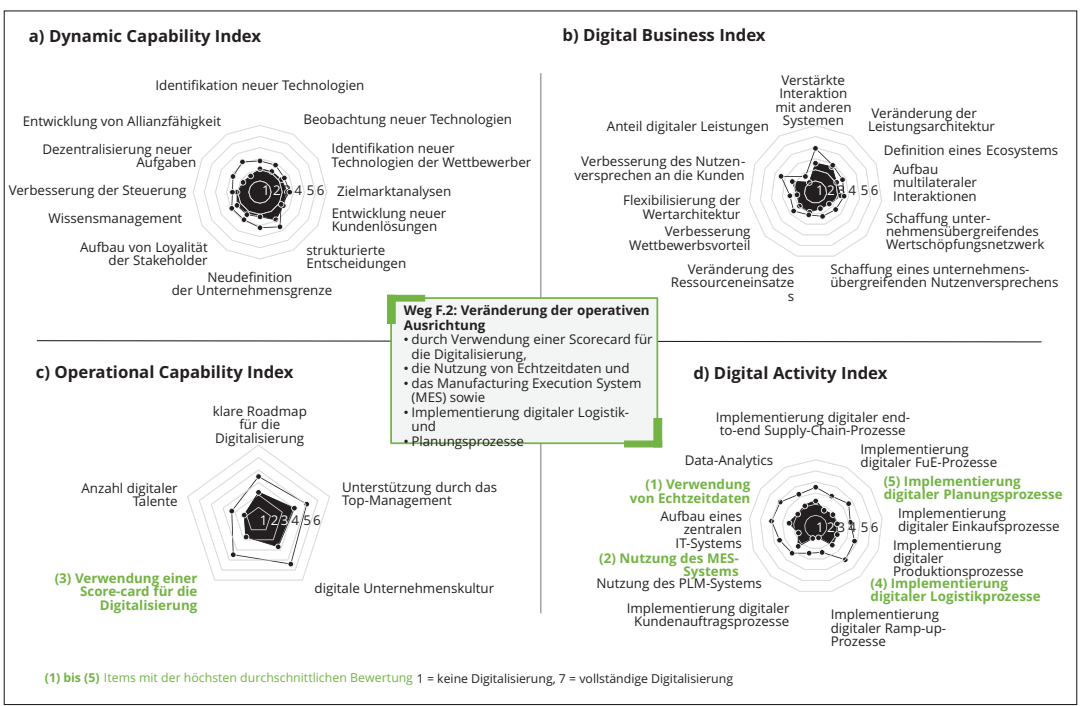

Abb.6.8 Weg (F.2) der digitalen Folger zu den digitalen Optimierern. (Quelle: eigener Entwurf) 
Die operative Verbesserung (Weg F.2) ist für die digitalen Folger einfacher als eine strategische Verbesserung (Weg F.1), weil sie dabei die ihnen bekannten, bislang isolierten Use-Cases und digitalen Pilotanwendungen verknüpfen können. Der Sprung von dort zur nächsten Stufe der digitalen Reife, d. h. zu den digitalen Potenzialträgern, ist aber größer als über den Zwischenschritt der Innovatoren (d. h. nach Weg F.1).

Angesichts der großen Zahl digitaler Potenzialträger in der Stichprobe (35 Prozent) ist zu erwarten, dass viele digitale Folger versuchen werden, direkt zu ihnen aufzuschließen. Ein solcher großer Sprung von den digitalen Folgern zu den digitalen Potenzialträgern (Weg F.3 von einem Digital Maturity Index von 3,04 bei den Folgern auf 5,06 bei den Potenzialträgern) erfordert eine klar verbesserte strategische und operative Ausrichtung auf die Digitalisierung, wenn auch nicht ganz so stark wie bei Nachzüglern, die noch weiter springen müssen, um zu den Potenzialträgern aufzuschließen (N.4). Entsprechend zeigen sich große Abweichungen zwischen den Rändern der schwarzen Ausgangsfläche als dem Status quo der digitalen Folger und dem äußeren Datenkreis als den Zielwerten der digitalen Potenzialträger in Abb. 6.9.

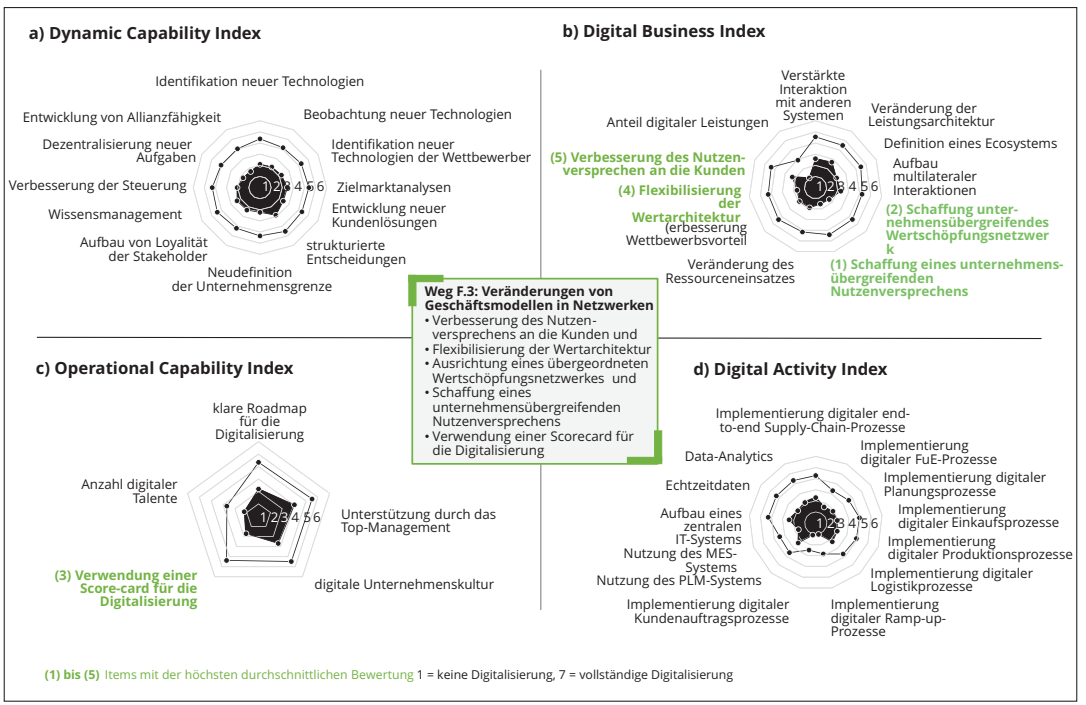

Abb.6.9 Weg (F.3) von den digitalen Folgern zu den digitalen Potenzialträgern. (Quelle: eigener Entwurf) 
Die fünf stärksten Abweichungen zwischen beiden Archetypen (grün gedruckt in Abb. 6.9) bieten jedoch kein klares Bild darüber, mit welchen Maßnahmen die digitalen Folger ansetzen sollen, um zu den digitalen Potenzialträgern aufzuschließen. Danach müssten sie neben

- dem Aufbau von Kontrollfähigkeiten durch Verwendung einer digitalen Scorecard wie auf dem Weg F.1 ihre Geschäftsmodelle durch

- Verbesserung des Nutzenversprechens an die Kunden und

- Flexibilisierung der Wertarchitektur

verbessern und zudem gemeinsame, mehrwertschaffende Angebote mit Partnern in neuen Netzwerken (Ecosystems) schaffen durch

- Ausrichtung eines übergeordneten Wertschöpfungsnetzwerkes und

- Schaffung eines unternehmensübergreifenden Nutzenversprechens.

Dieses Ergebnis der empirischen Untersuchung lässt sich damit erklären, dass anders als bei den Nachzüglern, die zu den Potenzialträgern nur aufschließen können, wenn sie zunächst ihre digitalen Fähigkeiten entwickeln oder aktvieren -, die digitalen Folger zumindest schon ansatzweise digitale Fähigkeiten entwickelt haben. Sie müssen gleichermaßen ihre strategische und operative Ausrichtung auf die Digitalisierung verbessern, was die Abweichungen zwischen den Rändern der schwarzen Ausgangsfläche als Status quo der digitalen Folger und dem äußeren Datenkreis als den Zielwerten der digitalen Potenzialträger in Abb. 6.9 verdeutlichen. Vier der fünf stärksten Abweichungen und damit Ansatzpunkte für Veränderungen sind jedoch Elemente von Geschäftsmodellen in Netzwerken.

Ein Sprung direkt von den digitalen Folgern zu den digitalen Champions ist dagegen wenig realistisch. Er wird immer mit einem Zwischenschritt zu den Potenzialträgern und damit über den Weg F.3 erfolgen.

\section{(3) Mögliche Wege der digitalen Optimierer zur Erhöhung der digitalen Reife}

Digitale Optimierer (Archetyp 4) haben sich im Zuge der Digitalisierung bislang stärker auf die Transformation ihrer Technologien und Prozesse sowie ihrer operativen Fähigkeiten gerichtet. Um die Chancen der Digitalisierung besser zu nutzen, müssen sie künftig ihre Geschäftsmodelle und Angebote stärker digitalisieren und mehr Veränderungsfähigkeiten aktivieren. Damit können sie

- zu den digitalen Potenzialträgern, d. h. Archetyp 2, aufschließen (Weg O.1),

- bei starken Anstrengungen sogar zu den digitalen Champions, d. h. zu Archetyp 1 (Weg O.2).

Die beiden möglichen Wege zur stärkeren digitalen Reife der digitalen Optimierer zeigt Abb. 6.10. 


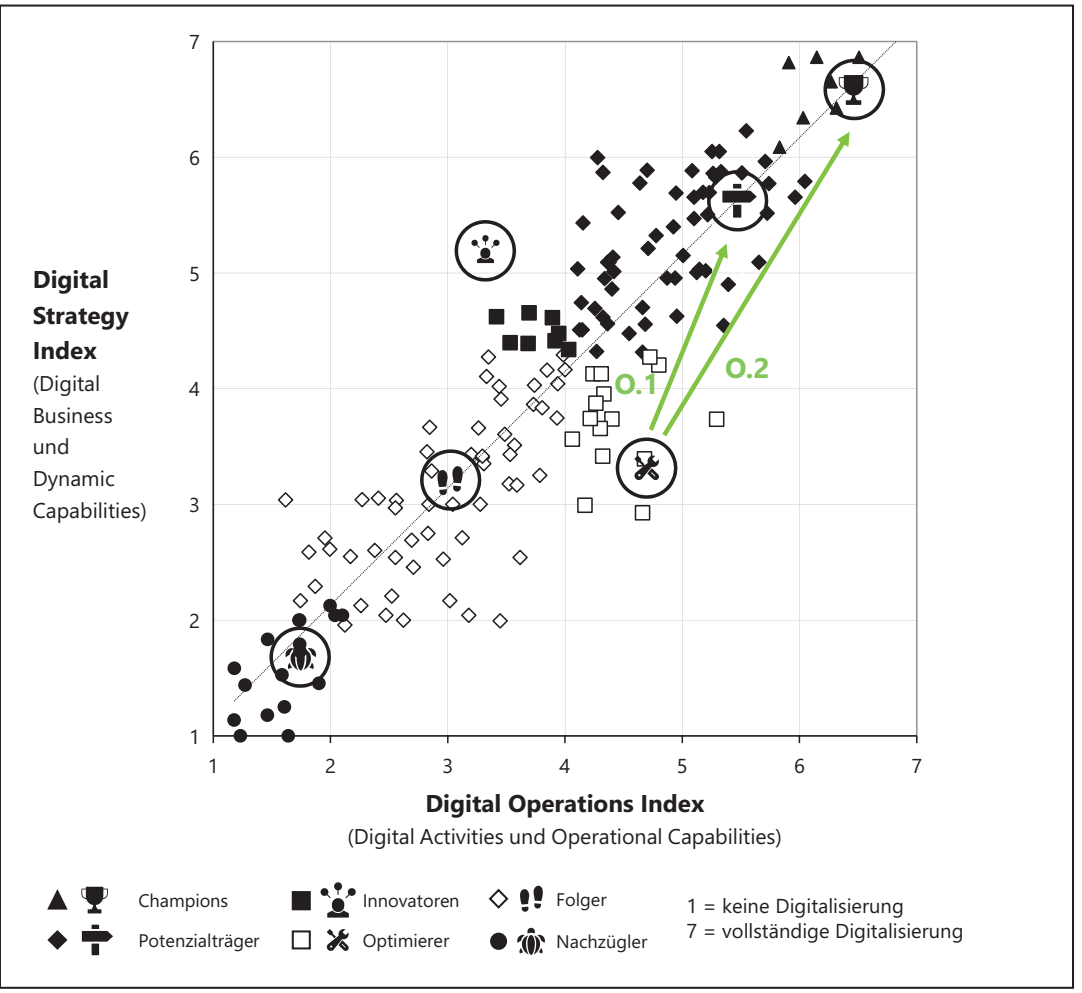

Abb. 6.10 Wege (O.1 und O.2) der digitalen Optimierer zur Erhöhung der digitalen Reife. (Quelle: eigener Entwurf)

Der Weg O.1 vom digitalen Optimierer zum digitalen Potenzialträger bedeutet, wie in Abb. 6.10 dargestellt, eine fast ausschließliche Veränderung in der strategischen Ausrichtung auf die Digitalisierung. Das sieht man an den deutlich größeren Unterschieden beim Dynamic Capability Index und beim Digital Business Index als beim Operational Capability Index und beim Digital Activity Index zwischen den Optimierern und Potenzialträgern in Abb. 6.11. Es zeigt sich aber auch an den fünf stärksten Abweichungen zwischen beiden Archetypen (grün gedruckt in Abb. 6.11) als Hinweis auf Maßnahmen, bei denen die digitalen Optimierer ansetzen sollten, um zu den digitalen Potenzialträgern aufzuschließen: Sie betreffen alle die strategische Ausrichtung auf die Digitalisierung. Noch einseitiger als die Folger beim Sprung zu den Potenzialträgern (Weg F.4) gilt es hier, 
- die Fähigkeit zu verbessern, neue Kundenlösungen zu entwickeln, aber auch

- durch Veränderung des Ressourceneinsatzes und

- Flexibilisierung der Wertarchitektur.

Geschäftsmodelle zu verbessern sowie gemeinsame, mehrwertschaffende Angebote mit Partnern in neuen Netzwerken (Ecosystems) zu schaffen durch

- Ausrichtung eines übergeordneten Wertschöpfungsnetzwerkes und

- Schaffung eines unternehmensübergreifenden Nutzenversprechens.

Eine derart umfassende strategische Ausrichtung ist in operativ fokussierten und u. a. Prozesse optimierenden Unternehmen nicht einfach.

Der Sprung $\mathrm{O} .2$ vom digitalen Optimierer zum digitalen Champion ist noch schwieriger und wird realistischerweise vorwiegend mit einem Zwischenschritt über die digitalen Potenzialträger erfolgen, sodass sich hier an den Weg O.1 der Weg P.1 von den digitalen Potenzialträgern zu den digitalen Champions anschließt, der in Punkt (5) betrachtet wird.

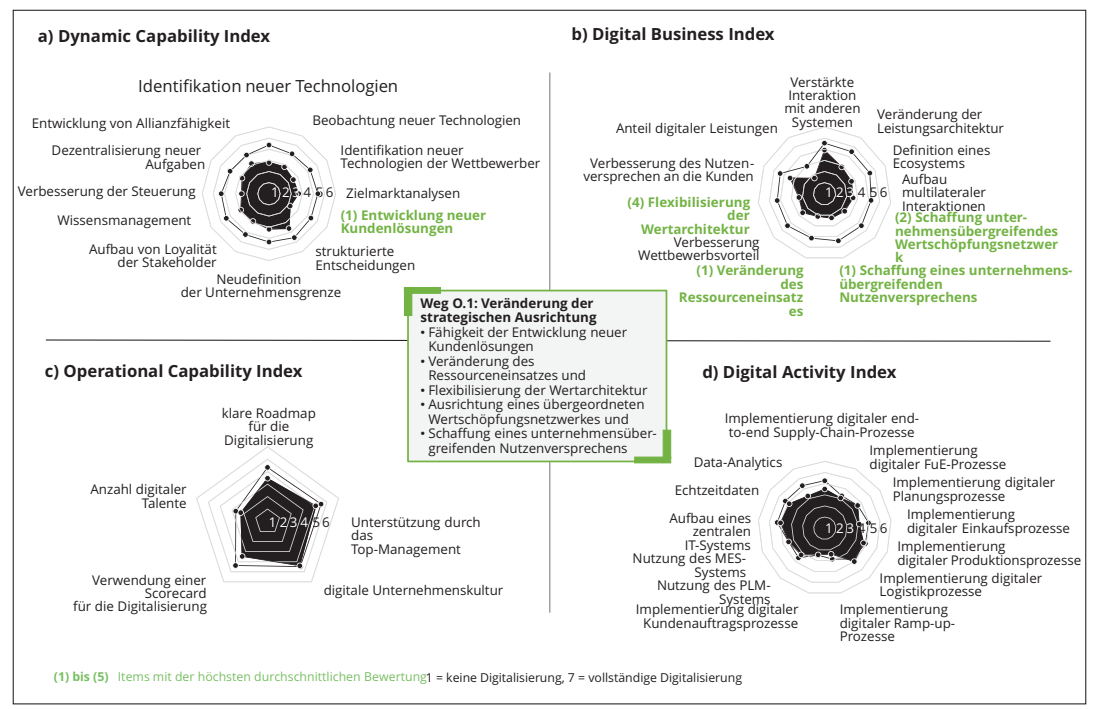

Abb.6.11 Weg O.1 von den digitalen Optimierern zu den digitalen Potenzialträgern. (Quelle: eigener Entwurf) 
(4) Mögliche Wege der digitalen Innovatoren zur Erhöhung der digitalen Reife

Digitale Innovatoren (Archetyp 3) haben sich im Zuge der Digitalisierung bislang stärker auf die strategische Transformation ihrer Veränderungsfähigkeiten sowie ihrer Angebote und Geschäftsmodelle gerichtet. Um die Chancen der Digitalisierung besser nutzen zu können, müssen sie künftig auch ihre Technologien und Prozesse stärker digitalisieren und operative digitale Fähigkeiten entwickeln. Damit können sie zu den digitalen Potenzialträgern, d. h. Archetyp 2, aufschließen (Weg I.1), bei starken Anstrengungen sogar zu den digitalen Champions, d. h. zu Archetyp 1 (Weg I.2) Die beiden möglichen Wege zur stärkeren digitalen Reife der digitalen Innovatoren zeigt Abb. 6.12.

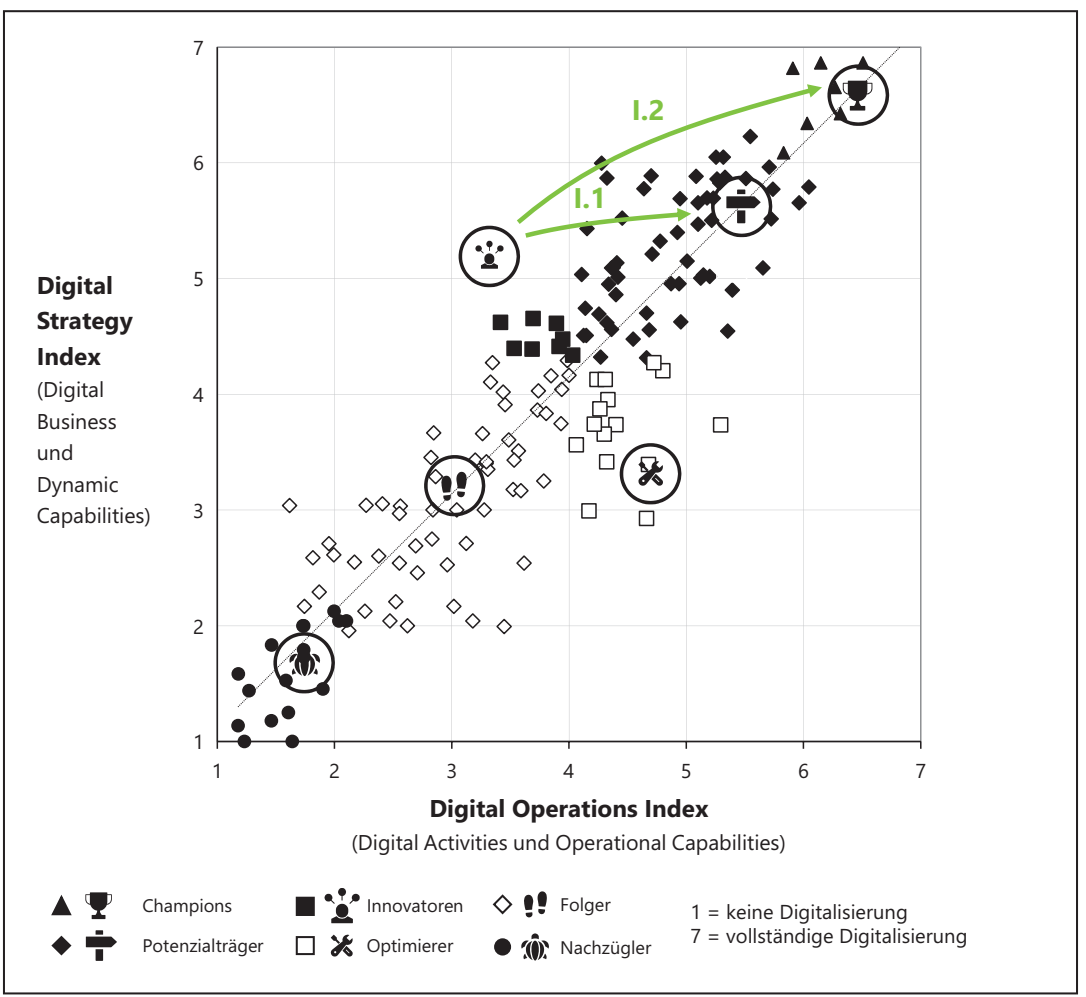

Abb. 6.12 Wege (I.1 und I.2) der digitalen Innovatoren zur Erhöhung der digitalen Reife. (Quelle: eigener Entwurf) 
Die deutlich größeren Unterschiede zwischen digitalen Innovatoren und digitalen Potenzialträgern beim Operational Capability Index und vor allem beim Digital Activity Index im Vergleich $\mathrm{zu}$ den beiden strategiebezogenen Teilindizes (Dyamic Capability Index und Digital Business Index) in Abb. 6.13 unterstreichen die Notwendigkeit einer stärker operativen Ausrichtung der digitalen Innovatoren. Die fünf deutlichsten Abweichungen der Innovatoren von den Potenzialträgern (in Abb. 6.13 grün gedruckt) bieten darüber hinaus Hinweise auf operative Maßnahmen, bei denen digitale Innovatoren ansetzen sollten, um zu den digitalen Potenzialträgern aufzuschließen, nämlich

- Aufbau eines zentralen IT-Systems,

- Verwendung von Echtzeitdaten und

- Nutzung eines Product Lifecycle Managements (PLM) zur Integration aller Informationen, die über den Lebenszyklus eines Produktes anfallen sowie

- Implementierung digitaler Einkaufprozesse, ergänzt um

- ein Wissensmanagement als wichtige Veränderungsfähigkeit zur NeuKonfiguration der operativen Fähigkeiten hinter Technologie- und Prozessverbesserungen.

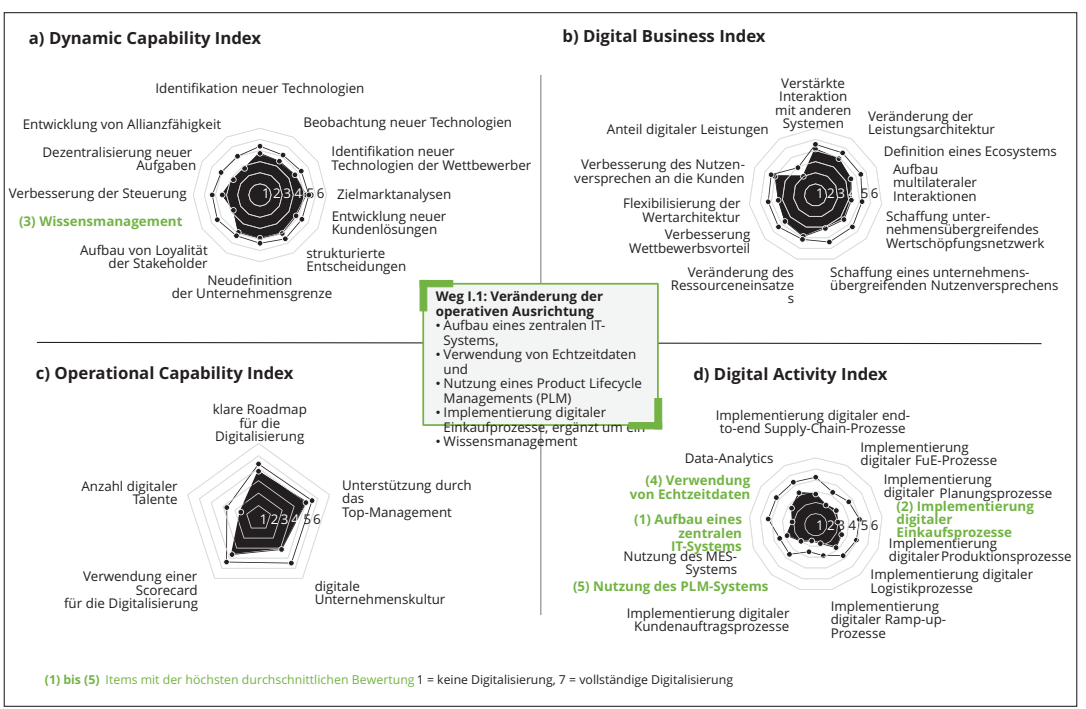

Abb.6.13 Weg (I.1) von den digitalen Innovatoren zu den digitalen Potenzialträgern. (Quelle: eigener Entwurf) 
Dieser Weg ist nicht einfach, weil ein innovatives Unternehmen, das bislang v. a. auf Innovationen in den Geschäftsmodellen für die Kunden am Markt setzt, sich in der betrieblichen Realität schwertut, nun zusätzlich interne operative Themen zu treiben.

Der Sprung I.2 vom digitalen Innovator zum digitalen Champion ist noch schwieriger und wird wie der Sprung O.2 der digitalen Optimierer nur mit einem Zwischenschritt über die digitalen Potenzialträger erfolgen. Deshalb schließt sich hier an den Weg I.1 der Weg P.1 der digitalen Potenzialträger zu den digitalen Champions an, der nun betrachtet wird.

\section{(5) Möglicher Weg der digitalen Potenzialträger zur Erhöhung der digitalen Reife}

Digitale Potenzialträger (Archetyp 2) schließlich haben bereits heute eine hohe digitale Reife bei strategischen und operativen Leistungen und Kompetenzen erreicht und nur noch den Weg P.1 zu den Digital Champions (Archetyp 1) zu managen (vgl. Abb. 6.14).

Für die große Gruppe der Potenzialträger (35 Prozent der Stichprobe) bedeutet der Weg zu den digitalen Champions eine weitere gleichmäßige Verstärkung ihrer strategischen und operativen Ausrichtung auf die Digitalisierung, da der Digital Maturity Index von einem durchschnittlichen Wert von 5,08 auf einen Wert von 6,44 auf einer 7er-Skala zu bringen ist. Entsprechend sind die Abweichungen zwischen den Rändern der schwarzen Ausgangsfläche als dem Status quo der digitalen Potenzialträger und dem äußeren Datenkreis als den Zielwerten der digitalen Champions in Abb. 6.15 für alle Teilindizes ähnlich groß. Die fünf stärksten Abweichungen (in Abb. 6.15 grün gedruckt) zeigen zudem, dass Potenzialträger zur Erhöhung ihrer digitalen Reife 


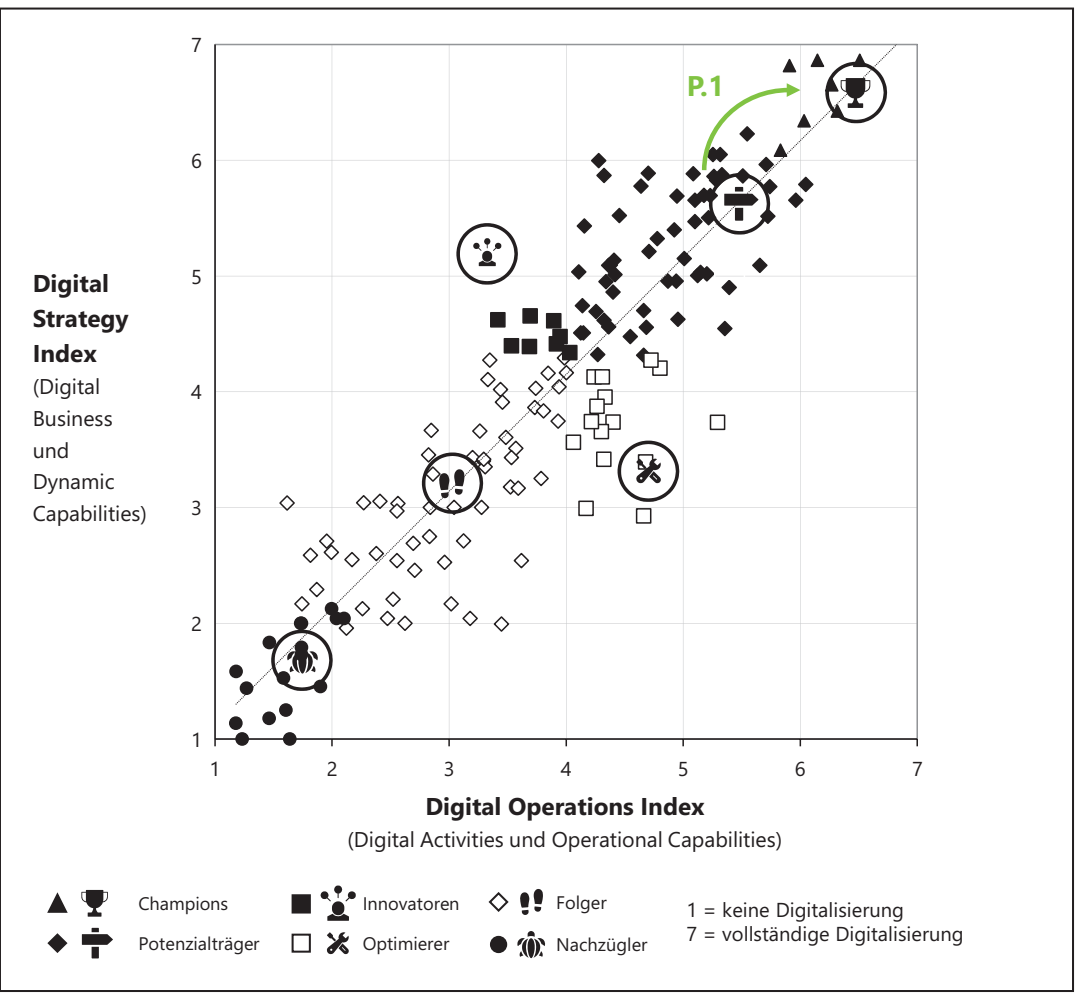

Abb.6.14 Weg (P.1) der digitalen Potenzialträger zur Erhöhung der digitalen Reife. (Quelle: eigener Entwurf)

- strukturierte Entscheidungen

- mit Unterstützung durch das Top-Management brauchen, die die digitalen Angebote weiter verbessern durch

- verstärkte Interaktion der Angebote mit denen anderer Systeme,

- Veränderung der Leistungsarchitektur,

aber auch

- die Definition neuartiger mehrwertschaffender Netzwerke (Ecosystems). 


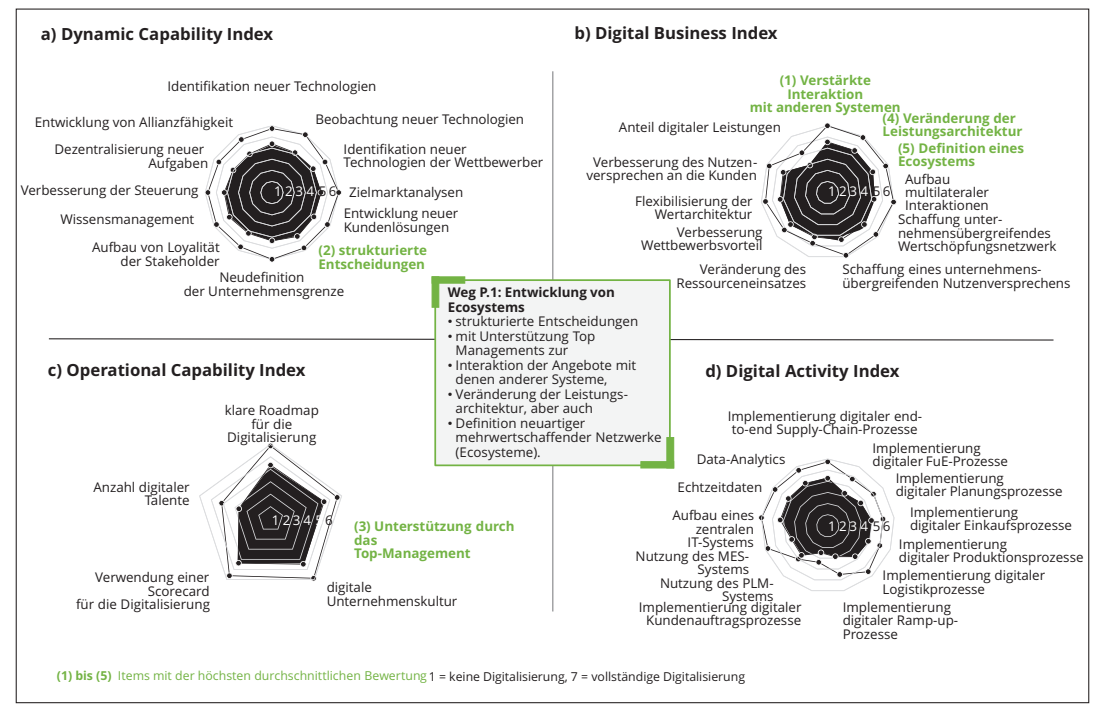

Abb.6.15 Weg (P.1) von den digitalen Potenzialträgern zu den digitalen Champions. (Quelle: eigener Entwurf)

Damit ist auch der Weg P1 der Potenzialträger zu den Champions anspruchsvoll, was noch einmal verdeutlicht, wie herausfordernd die Wege I.2 oder O.2 gewesen wären.

Die Ergebnisse unserer Befragung bei 160 deutschen Unternehmen zeigen damit Wege zur Erhöhung ihrer digitalen Reife, die als Ansätze einer individuellen Ausgestaltung des digitalen Transformationspfades eines jeden Unternehmens zu verstehen sind.

Ergänzend könnte die Frage gestellt werden, ob die digitale Transformation eher über ,greefields“ oder über ,brownfields“ erfolgen sollte (vgl. das fünfte Video). Bei ,greenfield“-Projekten wird eine völlig digitalisierte Fabrik auf einer grünen Wiese geplant und gebaut, bei ,brownnfield“-Anlagen sind bereits gebaut und in Betrieb. Weil der Wettbewerbsdruck steigt, was wir im nächsten Abschn. 6.2 begründen werden, ist eine weitere Beschleunigung der digitalen Transformation unumgänglich. Dafür müssen Ansatzpunkte gefunden werden (Abschn. 6.3 bis 6.6) (vgl. auch das folgende Video). 
Video 5: Fünf Wege

digitaler Reife -

„greenfileds“ und

„brownfields“ (https://doi.

org/10.1007/000-0t0)

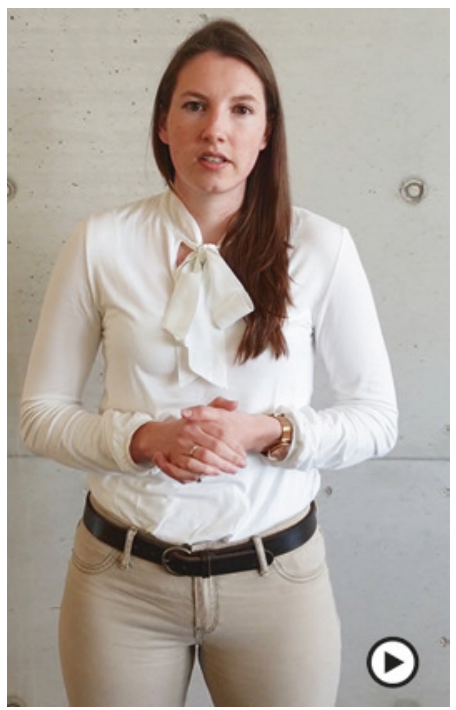

\subsection{Notwendigkeit einer weiteren Beschleunigung der digitalen Transformation}

In Abschn. 6.1 wurden unterschiedliche Wege zur Erhöhung der digitalen Reife aufgezeigt, mit denen sich die Chancen der Digitalisierung besser nutzen lassen und eine positive Gewinnwirkung möglich wird. Da der Wettbewerb weiter zunehmen wird, sollten Unternehmen dringend versuchen, ihre digitale Transformation zu beschleunigen, um in ihrem Markt konkurrenzfähig zu bleiben.

Volkswirtschaftlich lässt sich zeigen, dass die in Kap. 2 begründeten Digitalisierungspotenziale mit der Zeit kleiner werden. Deshalb reicht es nicht, wenn Unternehmen die in Abschn. 6.1 aufgezeigten Wege systematisch beschreiten, sie müssen gleichzeitig versuchen, dabei schneller zu sein als die Wettbewerber. Ähnlich wie in den Zeiten verstärkter Globalisierung in den 1990er Jahren wird es zu einem Wettlauf um eine möglichst frühzeitige Nutzung der Chancen der Digitalisierung kommen. Dieses Rennen beginnt spätestens dann, wenn die ersten Wettbewerber damit anfangen, digitale Gewinnpotenziale an den Markt weiterzugeben, um entweder eine bessere Marktposition zu erreichen oder Wettbewerbern zu schaden. Deshalb haben, wie in Abschn. 5.3 gezeigt, in derselben Branche Unternehmen, die dem Archetyp der Champions 
zugeordnet wurden, gegenüber den Nachzüglern deutliche Gewinn- und Umsatzvorteile durch die Digitalisierung, die für eine bessere Marktbearbeitung eingesetzt werden können.

Diese Wettbewerbsbeziehung wird in der Volkswirtschaftslehre idealtypisch mit der Edgeworth-Box (Abb. 6.16) veranschaulicht. Diese setzt bei den Chancen der Digitalisierung an, die sich für ein Unternehmen dadurch ergeben, dass bei Verringerung der Zielkonflikte zwischen Effizienz und Flexibilität infolge sinkender Transaktionskosten im traditionellen Geschäft größere Handlungsspielräume möglich werden, vgl. die Erklärungen in Kap. 2, Abb. 2.6. Dort wurde zunächst begründet, dass sich die Gewinn- bzw. Profitkurven $\mathrm{p}$ des Unternehmens durch die Nutzung von Plattformen und Gleichteilen von $\mathrm{p}_{0}$ auf $\mathrm{p}_{1}$ erhöhen, weil einerseits Einsparpotenziale bei der Nutzung von Plattformen und Gleichteilen in der Produktion und gleichzeitig Differenzierungsmöglichkeiten in Marketing und Vertrieb möglich werden (und durch neue Kombination der Gleichteile auch Innovationspotenziale geschaffen werden), was die Zielkonflikte verringert. Darüber hinaus wurde dargestellt, dass die Standardisierung von Schnittstellen im

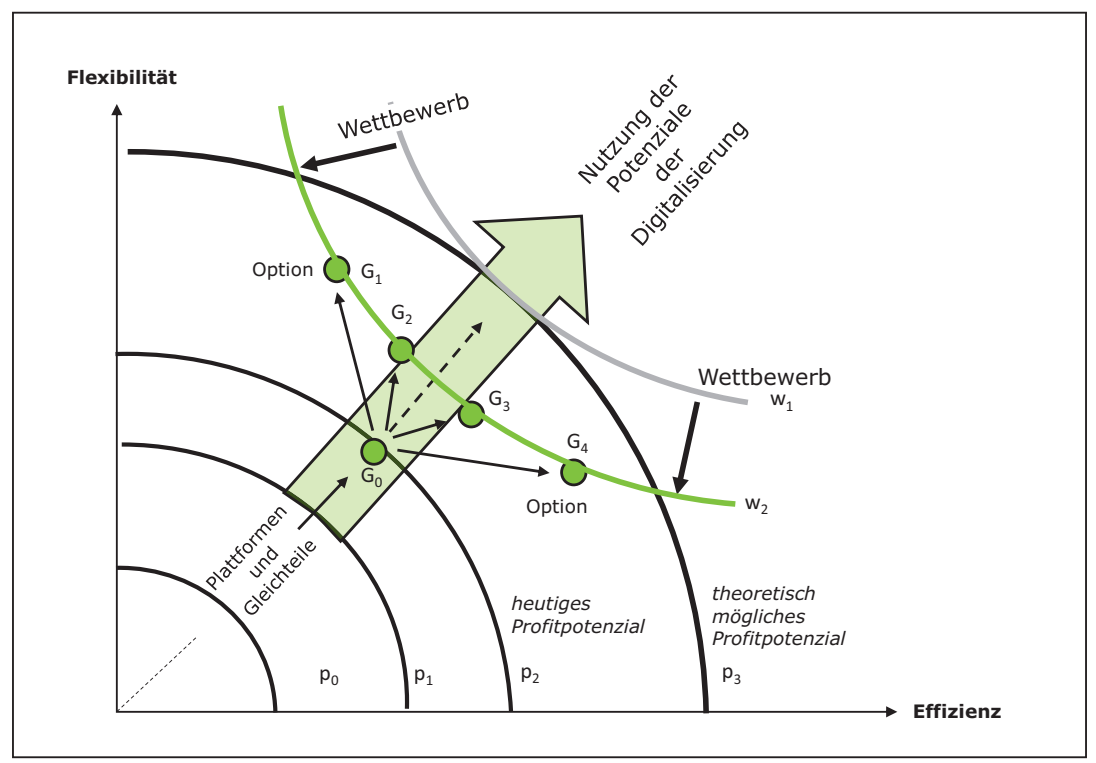

Abb.6.16 Verhandlungsraum infolge eines beschleunigten Wettbewerbs durch die Digitalisierung - Edgeworth Box. (Quelle: eigener Entwurf nach Franke 1985, S. 82) 
Zuge der Digitalisierung den Verhandlungsraum noch weiter vergrößert, wodurch sich die Gewinnkurve von $\mathrm{p}_{1}$ auf heute $\mathrm{p}_{2}$ verschoben hat.

Durch Nutzung der Chancen der Digitalisierung ist sogar eine Gewinnkurve $\mathrm{p}_{3}$ möglich. Allerdings werden die Wettbewerber ihrerseits versuchen, die Chancen der Digitalisierung zu nutzen und dadurch ihre Gewinnkurve von $\mathrm{w}_{1}$ auf $\mathrm{w}_{2} \mathrm{zu}$ erhöhen. Das schränkt dann die Handlungsoptionen des eigenen Unternehmens ein und verringert die möglichen Gewinnpotenziale vermutlich auf einen der neuen Gleichgewichtspunkte $\left(G_{1}\right.$ bis $G_{4}$ auf $w_{2}$ zwischen $p_{2}$ und $p_{3}$ in Abb. 6.16). Damit wird deutlich, dass Geschwindigkeit eine wichtige Rolle spielt, da die positiven ökonomischen Wirkungen der Digitalisierung eine zeitliche Dimension aufweisen: Der eigene Anteil am Gewinnpotenzial ist umso größer, je schneller die eigene digitale Transformation erfolgt, da dann im Idealfall einer sehr langen Zeit ohne Wettbewerberreaktionen die vollen ökonomischen Renten erwirtschaftet werden können. Ziel muss es deshalb sein, Zeitmonopole zu nutzen, um ein Image und eine Marktposition zu etablieren sowie Gelder hierfür die nächste Generation von (digitalen) Leistungen zu erwirtschaften.

$\mathrm{Zu}$ den Wettbewerbern gehören künftig auch immer mehr branchenfremde Konkurrenten, die mit neuen Geschäftsmodellen in etablierte Märkte eindringen und dort die Wettbewerbsintensität erhöhen (vgl. Abschn. 4.1).

Erstaunlich ist, dass dieser volkswirtschaftlich belegbare Effekt von vielen Unternehmen, die die Deloitte Digital Factory besucht und teilweise auch Workshops mitgemacht haben, bislang wenig gesehen wird. Der Wettbewerb wird deutlich unterschätzt und man vertraut implizit auf die hohe Anpassungsfähigkeit des eigenen Unternehmens. Bei vielen hat die Umstellung auf Lean Production sowie auf die Kombination von Mechanik und Elektronik (Mechatronik) in der FuE recht gut geklappt und sie gewinnen daraus den Optimismus, auch mit der digitalen Transformation klarzukommen. Bei dieser Argumentation wird jedoch übersehen, dass die digitale Transformation eben nicht nur einen unternehmerischen Funktionsbereich, z. B. Produktion oder FuE, betrifft, sondern alle Funktionsbereiche, die zur Leistungserstellung beitragen und neue Kompetenzen brauchen.

Deshalb sollen im Folgenden vier Ansatzpunkte zur weiteren Beschleunigung der Digitalisierung aufgezeigt werden, die sich aus vielen Gesprächen und Workshops mit Unternehmen in der Deloitte Digital Factory, am Lehrstuhl für Internationales Automobilmanagement der Universität Duisburg-Essen und beim jährlich in Duisburg stattfindenden Wissenschaftsforum Mobilität ergeben haben. ${ }^{6}$ Sie sind unabhängig vom Status quo der Digitalisierung, d. h. von den Archetypen digitaler Unternehmen, denen ein Unternehmen zugeordnet werden 
kann (Abschn. 5.3) und unabhängig vom Weg der digitalen Transformation, den es wählt (Abschn. 6.1). Es gilt,

- Leistungen über geeignete digitale Schlüssel zu vernetzen und zu skalieren (Accelerator of Digitalization (1), Abschnitt 6.3),

- Einspar- und Erlöspotenziale der Digitalisierung rechtzeitig zu quantifizieren (Accelerator of Digitalization (2), Abschn. 6.4),

- digitale Fähigkeiten durch (hybride) Agilität in der Organisation umzusetzen (Accelerator of Digitalization (3), Abschnitt 6.5) und

- Schutz vor ungewolltem Datenabfluss (Cyber Security) aufzubauen (Accelerator of Digitalization (4), Abschn. 6.6).

\subsection{Accelerator of Digitalization (1): Leistungen mithilfe geeigneter digitaler Schlüssel vernetzen und skalieren}

Oft geht die digitale Transformation nicht richtig voran, weil gerade die für unser Digital Maturity Assessment untersuchten größeren Unternehmen komplexe, oft durch Kooperationen und Akquisitionen zusammengebrachte Strukturen aufweisen, in denen an vielen Stellen einzelne, oft unverbundene Pilotanwendungen digitaler Technologien bei Prozessen, Angeboten und Geschäftsmodellen getestet werden. Deshalb wird ein erster Beschleuniger der digitalen Transformation darin gesehen, Leistungen zu vernetzen und zu skalieren, was über geeignete digitale Schlüssel möglich ist, die das digitale Potenzial (unternehmensweit) öffnen. Das soll hier am Beispiel des Einsatzes digitaler Technologien in Fertigungsprozessen gezeigt werden.

In Unternehmen werden heute häufig bereits in der Produktion für verschiedene Fertigungsarten wie Entwicklung auf Bestellung, Stapelverarbeitung, Fließbandfertigung, oder Durchlauffertigung ${ }^{7}$ unterschiedliche digitale Technologien eingesetzt: ein automatisierter Workflow, Sensoren und das Internet der Dinge, Analytiken, Augmented und Virtual Reality (VR/AR), digitale Zwillinge als digitale Repräsentanz eines materiellen oder immateriellen Objekts oder Prozesses aus der realen Welt und ein digitales Rückgrat. Dabei nehmen vom automatisierten Workflow zum digitalen Rückgrat das Ausmaß der digitalen Technologien zu und die Flexibilität der Unternehmen ab, weshalb sich die einzelnen Technologien für die Fertigungsprozesse unterschiedlich gut eignen. Abb. 6.17a visualisiert die Komplexität derartiger Digitalisierungsbemühungen. Sie ist in Zusammenarbeit mit einem großen Elektrotechnik- und Elektronikunternehmer in der Deloitte Digital Factory entstanden, gilt aber auch unabhängig 
davon. Das Unternehmen ist an 200 Standorten weltweit tätig; es gibt jeweils in unterschiedlichen Werken alle vier Fertigungsarten und alle digitalen Technologien in bislang isolierten Use-Cases irgendwo auf der Welt. Das liegt daran, dass das Unternehmen durch Akquisitionen stark gewachsen ist und jedes neu akquirierte Unternehmen unterschiedliche (IT-)Systeme mitgebracht hat, die nie konsolidiert wurden. Auch gibt es keine wirklich zentrale Koordination der IT und der Produktionsprozesse. Dadurch sind in dem Unternehmen 42 isolierte IoT-Piloten über alle Fertigungsprozesse hinweg entstanden. Viel Geld wurde wenig zielführend investiert, entsprechend groß war die Unzufriedenheit in der Organisation.

Die digitale Transformation der Fertigungsprozesse sollte überall dort getrieben werden, wo sich die digitalen Prozesse in den verschiedenen Tochtergesellschaften weltweit dafür gut eignen. Deshalb wurde eine gute, mittlere und geringe Eignung der einzelnen Fertigungsprozesse mithilfe von Teilnehmern an Workshops in der Deloitte Digital Factory abgeschätzt (vgl. die Bewertung „+++" bis „, “ in Abb. 6.17). Danach scheint eine echte Beschleunigung der digitalen Transformation der Fertigungsprozesse nur möglich, wenn einzelne Pilotanwendungen gemeinsam als ,digitale Schlüssel“ definiert werden, die über alle Fertigungsprozesse, aber auch über Softwaregrenzen und Firewalls hinweg weltweit eingesetzt, untereinander vernetzt und skaliert werden und damit der digitalen Transformation „den Weg öffnen“. Solche Schlüssel zur beschleunigten Digitalisierung der Fertigungsprozesse müssen zunächst an einer Stelle umfassend getestet werden und dann im Schneeballprinzip als vernetzte Schlüssel auf ähnliche Fertigungsprozesse und schließlich als unternehmensweite Schlüssel auf alle Fertigungsprozesse im ganzen Unternehmen angewendet werden (Abb. 6.17b).

Auch unser beispielhaft betrachtetes Elektrotechnik- und Elektronikunternehmen wollte die Vielzahl von Pilotanwendungen reduzieren und einzelne integrierte, unternehmensspezifische Digitalisierungsschlüssel auswählen, die zu den unternehmensspezifischen Gegebenheiten passen und einzelne bislang isolierte Pilotanwendungen im Unternehmen integrieren. Das Unternehmen wählte dafür Anwendungen im Produktionsprozess wie Wearables (kleine vernetzte Computerbrillen), RFID-Chips (die mit Radiowellen gespeicherte Informationen an ein Lesegerät übermitteln können) und MES, d. h. Produktionsleitsysteme. Sie waren in der gegebenen IT-Landschaft und im operativen Umfeld des Unternehmens leicht umsetzbar, konnten einfach zwischen den verschiedenen Standorten des Unternehmens ausgetauscht werden und gleichzeitig im Zeitablauf zusätzliche Use-Cases und Anwendungen integrieren, um damit eine zunehmende Vernetzung zu ermöglichen. 
a) unterschiedliche digitale Technologien für unterschiedliche Fertigungsprozesse

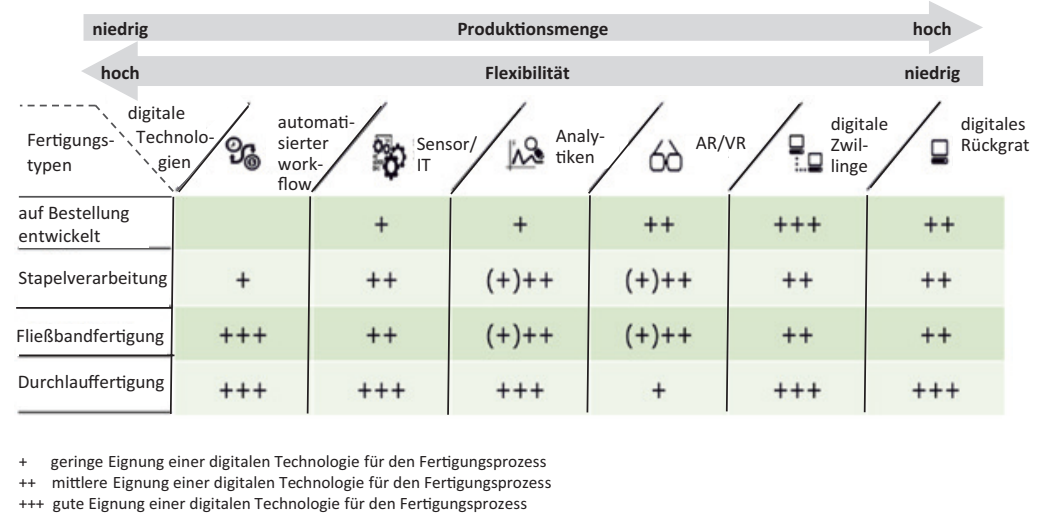

b) digitale Schlüssel: Vernetzung und Skalierung digitaler Piloten

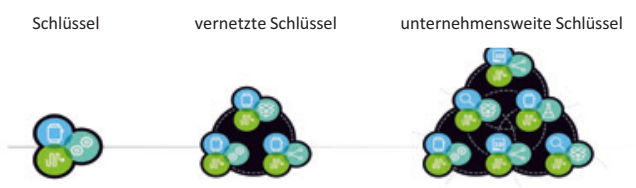

Abb. 6.17 Unterschiedliche digitale Technologien für unterschiedliche Fertigungsprozesse zur Beschleunigung der digitalen Transformation. (Quelle: eigener Entwurf)

Der Weg zu solchen Digitalisierungsschlüsseln ist allerdings steinig und viele Unternehmen kommen bisher noch nicht wirklich voran. Dies liegt daran, dass die zur Vernetzung und Erreichung von digitalen Prozessen, Produkten oder gar Geschäftsmodellen notwendige IT-Infrastruktur oft unzureichend ist, da sie auf die immensen Rechenleistungen und Bandbreiten des mobilen Datentransfers nicht eingerichtet ist und durch komplexe IT-Sicherheitsrichtlinien verzögert wird (vgl. auch Abschnitt 6.6). Selbst in der kleinen und überschaubaren Deloitte Digital Factory in Düsseldorf gab es zudem Managementprobleme auf dem Weg zu vernetzten Prozessen. Vor allem mangelnde Kommunikation und Abstimmung zwischen ITlern und Managern der übrigen Funktionsbereiche, die in ihren Bereichen gleichzeitig auch noch andere Aufgaben haben, führen häufig zu einseitig definierten Spezifikationen. Dies verursacht auf der einen Seite viel 
Arbeit und wird auf der anderen Seite nicht verstanden. Fehlende Transparenz und Führung, v. a. aber ein mangelndes Konfliktmanagement verstärken oft das Silodenken und Interessenskonflikte, die häufig unterschätzt werden.

Gespräche und Workshops in der Deloitte Digital Factory haben gezeigt, dass der tatsächliche Aufwand der IT-Lösungen von Nicht-IT-Experten klar unterschätzt wird. Für die acht Kernanwendungen der Produktionslinie in der kleinen Digital Factory in Düsseldorf mussten 300 Verbindungen über fünf Clouds geschaffen und zehn unterschiedliche Firewalls überwunden werden. Für eine 10-minütige Simulation wurden Daten insgesamt $1.500 .000 \mathrm{~km}$ weit, d. h. etwa zweimal zum Mond und zurück oder etwa 40 Mal um die Erde, geschickt.

Ein erster Ansatzpunkt zur Beschleunigung der digitalen Transformation ist also die unternehmensweite Vernetzung und Skalierung von Leistungen über geeignete digitale Schlüssel. Voraussetzung dafür ist eine neue Art der Zusammenarbeit von ITlern und Managern anderer Funktionsbereiche, um das Silodenken in diesen Abteilungen zu überwinden und die neuen digitalen Lösungen durch die Organisation zu treiben (vgl. das Video 6).

Video 6: Cross-

funktionale

Zusammenarbeit von

Business und IT (https://

doi.org/10.1007/000-0sz)

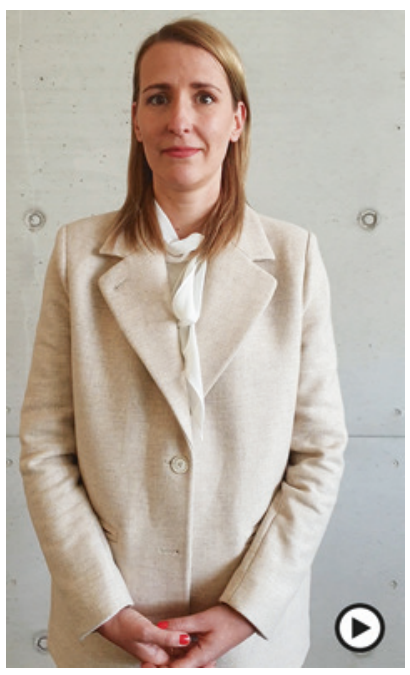


Dafür braucht es Transparenz und Führung, d. h. eine Unterstützung durch das Top-Management, die auch schon bei vielen der unterschiedlichen Wege in die digitale Transformation (Abschn. 6.1) als einer der fünf wichtigsten Ansatzpunkte genannt wurde. Erst wenn eine solche unternehmensweite Vernetzung und Skalierung digitaler Prozesse über ausgewählte digitale Schlüssel gelingt, kann die Digitalisierung der Angebote und Geschäftsmodelle auf die gleiche Weise folgen. Statt auf eine große, alle Probleme lösende, digitale Megaplattform zu warten, gilt es, die digitale Transformation mithilfe von Digitalisierungsschlüsseln nach und nach bei allen Leistungen zu beschleunigen und dabei auf dem eingeschlagenen Weg zu lernen.

\subsection{Accelerator of Digitalization (2): Einspar- und Erlöspotenziale durch die Digitalisierung rechtzeitig quantifizieren}

Zusätzlich zu einer Vernetzung und Skalierung der digitalen Leistungen mithilfe geeigneter digitaler Schlüssel, kann die digitale Transformation auch durch Quantifizierung möglicher Einspar- und Erlöspotenziale beschleunigt werden. Denn auch hier gilt: „Only what can be measured can be managed“. Auch wenn bei diskontinuierlichen Umfeldveränderungen wie durch die Digitalisierung traditionelle Kennzahlensysteme zu kurz greifen, brauchen Investoren angesichts der hohen Unsicherheit eine Quantifizierung der ökonomischen Auswirkungen der Digitalisierung, um z. B. unterschiedliche Investitionsoptionen zu priorisieren. Aber auch Manager benötigen eine solche Quantifizierung, um sie z. B. den Budgetvorgaben zugrunde zu legen, mit denen sie eine stärkere digitale Transformation der Wertschöpfung in ihrer Organisation voranbringen können.

Dabei sind die Kennzahlen und Budgets so zu wählen, dass - wie in Abschn. 6.3 dargestellt - die Implementierung der digitalen Transformation nicht allein den ITlern unter der Leitung des CIO überlassen wird. Denn die würden einen eher ,ITgetriebenen Implementierungsansatz" verfolgen, d. h. ein solides und standardisiertes IT-Rückgrat für die digitale Transformation schaffen, aber mit hohen Anfangsinvestitionen und zeitlich sehr späten positiven Effekten in den Funktionsbereichen. Es muss durch die Kennzahlen und Budgets aber auch verhindert werden, dass die Implementierung der digitalen Transformation allein dem Produktionsbereich unter der Leitung des COO überlassen wird, der häufig einen „Operations-getriebenen“ Implementierungsansatz verfolgt. Dann wären zwar die Anfangsinvestitionen geringer als bei einer IT-getriebenen Lösung. Es besteht jedoch die Gefahr, dass individuelle IT-Applikationen Priorität vor Gesamtlösungen erhalten und eine Konzentration auf Prozesse erfolgt, weniger auf Produkte und Geschäftsmodelle. 
Gespräche in der Deloitte Digital Factory in Düsseldorf und am Lehrstuhl für Internationales Automobilmanagement der Universität Duisburg-Essen haben gezeigt, dass eine „Top-down-Implementierung“ der digitalen Transformation erforderlich ist, die beim CEO angesiedelt sein sollte. Erforderlich ist eine Quantifizierung aller Einspar- und Erlöspotenziale durch die Digitalisierung bei unternehmensweiter Nutzung von Ressourcen, um zu verhindern, dass Investitionsmittel - wie so oft in großen Organisationen - in zu vielen Einzelinvestitionen aufgerieben werden. Im Folgenden soll am Beispiel von digitalen Prozessen gezeigt werden, wie sich die Kosten und Erlöse der digitalen Transformation quantifizieren lassen.

Durch die digitale Transformation der Prozesse sind vor allem Kosteneinsparungen in Fertigung, Logistik und Vertrieb, aber auch Erlössteigerungen möglich. Wie in Abschn. 6.3 soll hier die Quantifizierung von Kosteneinsparungen am Beispiel des Fertigungsprozesses des Elektro- und Elektronikunternehmens gezeigt werden. Bei Gesprächen und Workshops in der Digital Factory in Düsseldorf wurden hierzu vier wichtige Einsparpotenziale im Fertigungsprozess identifiziert, die durch die Digitalisierung möglich werden (vgl. Abb. 6.18):

1. verbesserte Anlageneffizienz: Bessere Kapazitätsauslastung, geringere Standzeiten und geringere Wechselkosten durch die Digitalisierung können die Kosten in der Fertigung um zusammen 15 bis 20 Prozent verringern.

2. verbesserte Qualität: Verringerter Ausschuss, bessere Materialausnutzung und schnellere Erreichung der optimalen Produktionsqualität beim Hochlauf durch die Digitalisierung können die Qualitätskosten in der Fertigung um zusammen 20 bis 50 Prozent reduzieren.

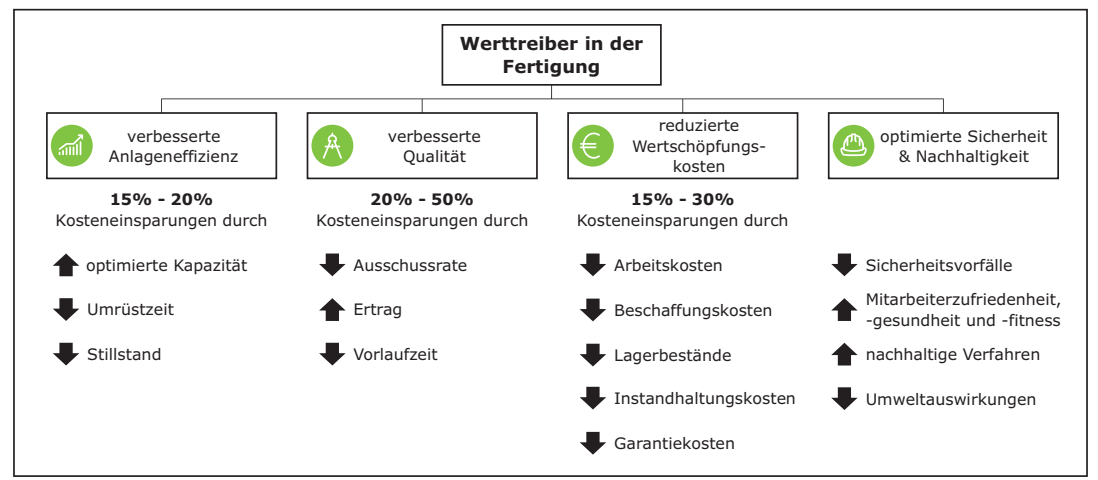

Abb.6.18 Quantifizierung der Einsparpotenziale in der Fertigung. (Quelle: Deloitte Digital Factory) 
3. reduzierte Wertschöpfungskosten: Geringere Lohn- und Beschaffungskosten, geringere Lagerhaltung und geringere Wartungs- und Garantiekosten durch die Digitalisierung können die Kosten in der Fertigung um zusammen weitere 15 bis 30 Prozent verringern. ${ }^{8}$

4. optimierte Sicherheit und Nachhaltigkeit: Eine sinkende Zahl von Unfällen, steigende/s Zufriedenheit, Gesundheit und Wohlergehen der Mitarbeiter, steigende Nachhaltigkeit und sinkende Umweltbelastung durch die Digitalisierung können die Fertigungskosten schließlich nochmals verbessern, ohne dass sich daraus unmittelbar eine Kostenwirkung ergibt.

Zur exakten Quantifizierung der Einsparpotenziale durch die Digitalisierung der (Fertigungs-)Prozesse ist ein dreistufiges Vorgehen nötig (vgl. Abb. 6.19):

1. Identifikation der Wirkungsbereiche der digitalen Transformation entlang der Dimensionen Arbeitsstationen, Funktionen und Kalenderjahr, z. B. die Verbesserung der Qualität in der Montage (Funktion) 2020 (Jahr) durch einen Montageroboter (Arbeitsstation), sowie dem größten Ansatzpunkt für Einsparpotenziale bei den Betriebskosten. Möglich sind direkte Einsparpotenziale z. B. durch Verringerung des Stundensatzes bei höherer Automatisierung der betroffenen Arbeitsstationen und indirekte Einsparpotenziale z. B. durch Verringerung der Mängel im ersten Produktionsdurchlauf und eine entsprechende Verringerung der Nachbearbeitungszeit und -kosten.

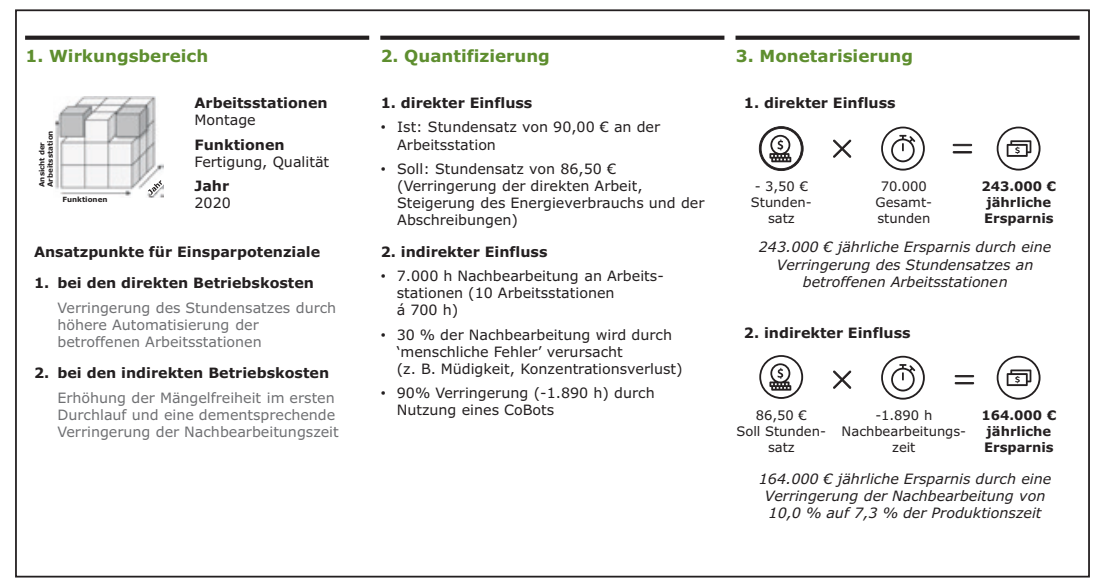

Abb. 6.19 Dreistufiger Prozess der Berechnung digitaler Prozessverbesserungen. (Quelle: Deloitte Digital Factory) 
2. Quantifizierung der direkten und indirekten Einsparpotenziale bei den Betriebskosten. Dafür muss z. B. der zusätzliche Investitionsaufwand eines Montageroboters gegen die Einsparungen bei menschlicher Arbeitszeit und die reduzierte Fehlerhäufigkeit aufgerechnet werden.

3. Monetarisierung der Einsparpotenziale, indem die direkten und indirekten Einsparpotenziale jeweils mit der Bedarfsmenge multipliziert werden, um einen Gesamteffekt zu bestimmen und Optionen der Verbesserung, z. B. für Digitalisierungsschlüssel (Abschn. 6.3) zu priorisieren.

Die Quantifizierung einzelner digitaler Anwendungen wie z. B. des Einsatzes eines Roboters alleine zeigt noch keine große Wirkung, da es sich im Grunde um ein traditionelles Vorgehen handelt. Deshalb bringt erst die Kombination verschiedener digitaler Anwendungen die digitale Transformation stärker voran, weshalb auch die Einspar- und Erlöspotenziale bei Interdependenzen zwischen den Anwendungen quantifiziert werden müssen. In Abb. 6.20a ist das Beispiel einer digitalen Fertigungszelle dargestellt, in der neben Montagerobotern (CoBots) auch 3D-Drucker, Radio-Frequency-Identification-(RFID-)Technologien, sensorbasierte Wartungstechnologien (Predictive Maintenance) und sensorbasierte Qualitätstechnologien (Predictive Quality) zum Einsatz kommen, zwischen denen wechselseitige Verbindungen bestehen. So können z. B. sensorbasierte Wartungstechnologien die Ausfallzeiten der Montageroboter dauerhaft reduzieren und ermöglichen sensorbasierte Qualitätstechnologien eine schnellere Anpassung der Montageprozesse. Die digitalen Qualitätstechnologien unterstützen wiederum die Wartungstechnologien, da sie Informationen über eine höhere Nachbearbeitungsrate weitergeben. Dadurch schafft die Kombination dieser fünf Technologien einen Mehrwert, der in einer Matrix (Abb. 6.20b) berechnet werden kann.

Die Wirkung von Interdependenzen bei der Vernetzung digitaler Prozesse kann die Wirkungen einzelner isolierter Prozesse teilweise um ein Vielfaches übertreffen. So hat z. B. ein großer deutscher Automobilzulieferer mit Unterstützung durch die Deloitte Digital Factory eine Fertigungslinie durchgängig an drei globalen Standorten in identischer Weise digitalisiert und erreichte damit zwei Jahre hintereinander Kosteneinsparungen von jeweils 30 Prozent, im zweiten Jahr v. a. durch gemeinsame Lerneffekte.

Die rechtzeitige Quantifizierung solcher Einsparpotenziale, aber auch z. B. von Erlöspotenzialen wie einer verbesserten Differenzierung durch digitale Prozesse, kann die digitale Transformation sehr stark beschleunigen. Nur dadurch können gerade in Zeiten hoher Unsicherheit Digitalisierungsoptionen priorisiert werden, um Wettbewerberreaktionen möglichst zuvorzukommen. 


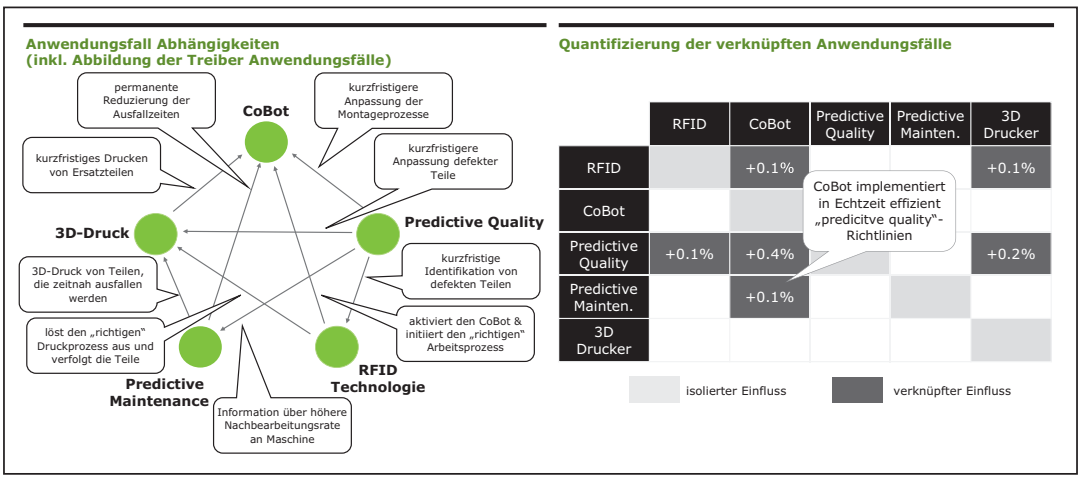

Abb. 6.20 Berechnung der Interdependenzen bei der Vernetzung digitaler Prozesse. (Quelle: Deloitte Digital Factory)

Wie bei der Vernetzung und Skalierung der Leistungen mithilfe digitaler Schlüssel (Abschn. 6.3) ist auch bei der Quantifizierung der Einspar- und Erlöspotenziale durch die Digitalisierung das Top-Management besonders gefordert, über Top-down-Vorgaben die Beschleunigung der digitalen Transformation zu treiben. Das ist keine einfache Aufgabe, da es in etablierten Unternehmen vielfältige Barrieren zu überwinden gilt, weil Schutzvorkehrungen gegen zu viel Wandel bestehen und weil eingeübte Managementprozesse Veränderungen verhindern. Hiergegen hilft es, durch kurze, bindende Entscheidungsprozesse erste Lösungen $\mathrm{zu}$ entwickeln und dadurch die digitale Transformation schrittweise, aber systematisch voranzutreiben (vgl. das nächste Abschn. 6.5).

\subsection{Accelerator of Digitalization (3): Digitale Fähigkeiten durch (hybride) Agilität in der Organisation umsetzen}

Um rasch mit der digitalen Transformation $\mathrm{zu}$ beginnen und auf dem eingeschlagenen Weg zu lernen, ist es wichtig, dass Unternehmen Veränderungsfähigkeiten (Dynamic Capabilities, vgl. Kap. 4) nicht nur aktivieren, sondern auch in der Organisation umsetzen. Sie brauchen damit nicht nur die Fähigkeiten, (1) die Umfeldveränderungen durch die Digitalisierung zu erkennen (Sensing), (2) neue strategische Optionen (Wege der digitalen Transformation) zu wählen (Seizing) und (3) die operativen Fähigkeiten entsprechend anzupassen (Reconfiguring), sondern sie müssen diese operativen Skills auch wirklich umsetzen. 
Dabei hilft in Zeiten von hoher Unsicherheit, wie gegenwärtig durch die Digitalisierung, die Fähigkeit der Agilität. ${ }^{9}$ Gemeint ist allerdings nicht nur „ITAgilität" ${ }^{\text {"10 }}$, sondern „organisationale Agilität“ als genau diese Fähigkeit der Umsetzung der Dynamic Capabilities in der Organisation. ${ }^{11}$ Organisationale Agilität erlaubt kurze, bindende Entscheidungsprozesse, die schrittweise den optimalen Weg bestimmen und von minimal lebensfähigen Produkten (Minimal Viable Products) ausgehen. ${ }^{12}$ Sie brechen eingefahrene, interne Prozesse auf, die die Transformationswege behindern. ${ }^{13}$

So wie Veränderungsfähigkeiten in Abhängigkeit von der Diskontinuität der technologischen Veränderungen unterschiedlich weitreichend sein können (,first“ and „second order" Dynamic Capabilities, vgl. Kap. 4), ist auch die Agilität umfeldabhängig, d. h. nicht in allen Branchen notwendig und auch nicht immer möglich: ${ }^{14}$ In wenig dynamischen Umfeldern ist Agilität nicht erforderlich und in kapitalintensiven Branchen nicht uneingeschränkt möglich, weil dort Effizienz notwendig ist, um die hohen fixen Investitionskosten zu verteilen. Dann ist es wichtig, den Übergang von der gegenwärtigen zur zukünftigen Existenz zu managen, ${ }^{15}$ das zeigen viele Gespräche in der Deloitte Digital Factory und am Lehrstuhl für Internationales Automobilmanagement der Universität DuisburgEssen. Dies gilt speziell in kapitalintensiven Branchen, in denen Unternehmen so lange die eigene Wertschöpfung in der alten Technologie halten möchten, bis es mit dem Durchbruch einer neuen Technologie gelingt, neues Wissen und eine signifikante neue eigene Wertschöpfung aufzubauen (Ramp-up) ${ }^{16}$. Dann erst möchten sie die Wertschöpfung in der traditionellen Technologie abbauen (Ramp-down). Bei hoher Unsicherheit in kapitalintensiven Branchen ist deshalb Ramp-down- und Ramp-up-Fähigkeit ${ }^{17}$, d. h. die Effizienzorientierung durch Optimierung von Größen- und Verbundvorteilen, ${ }^{18}$ erforderlich.

Damit besteht zwischen Agilität und Effizienz ein Zielkonflikt ähnlich dem zwischen Effizienz und Flexibilität (vgl. Kap. 2 und 6.2), der sich nicht ganz auflösen lässt: Agilität bedeutet auch Flexibilität, die der effizienzorientierten Fähigkeit der Optimierung von Größen- und Verbundvorteilen entgegensteht.

Gemäß den Erklärungen der Wirtschaftspolitik ${ }^{19}$ sind bei derart unüberwindbaren Differenzen Mediationslösungen zu suchen. Ein Ansatzpunkt zur Mediation des Konflikts zwischen Agilität und Effizienz, wird in Veränderungsfähigkeiten gesehen. ${ }^{20}$ Diese verbessern die Agilität zur Anpassung der Operational Capabilities bei Unsicherheit, weil z. B. die Veränderungsfähigkeit des Reconfiguring bewirkt, dass im Rahmen von Agilität die Produktentwicklung unter Unsicherheit schrittweise (iterativ) und inkrementell über Minimum Viable Products (MVP) geschaffen wird. Außerdem bewirkt die Veränderungsfähigkeit 
des Reconfiguring die schnelle Umsetzung von Feedback, das Lernen von den Kunden und den Aufbau einer Veränderungskultur. ${ }^{21}$ Veränderungsfähigkeiten minimieren aber gleichzeitig auch Effizienzverluste, d. h. Kosten der Erreichung eines bestimmten Maßes an organisationaler Agilität. ${ }^{22}$ Darüber hinaus können sie sogar die Effizienz verbessern. ${ }^{23}$ Z.B. bewirkt Sensing, dass im Rahmen der Optimierung von Größen und Verbundvorteilen der Auf- und Abbau von Wertschöpfung gesamthaft geplant wird, ${ }^{24}$ und legt das Reconfiguring fest, ob die Kapitalintensität nur kurzfristig erhöht oder langfristig reduziert wird und ob damit die Operational Capabilities verändert werden. ${ }^{25}$

In Erweiterung von Kap. 4 und insbesondere Abb. 4.1 und 4.3 bedeutet dies, dass in Zeiten der Digitalisierung mit hoher Unsicherheit drei Fähigkeiten wichtig sind:

1. Veränderungsfähigkeiten als Fähigkeit der strategischen Entscheidung über Veränderungen

2. Agilität und

3. Optimierung von Größen- und Verbundvorteilen.

Sie müssen zusammen gedacht werden (Abb. 6.21), weil die Veränderungsfähigkeiten des Sensing, Seizing und Reconfiguring den Rahmen dafür bieten, wie die Fähigkeiten der Agilität und der Optimierung von Größen und Verbundvorteilen zu handhaben sind. Dadurch kann die digitale Transformation beschleunigt werden.

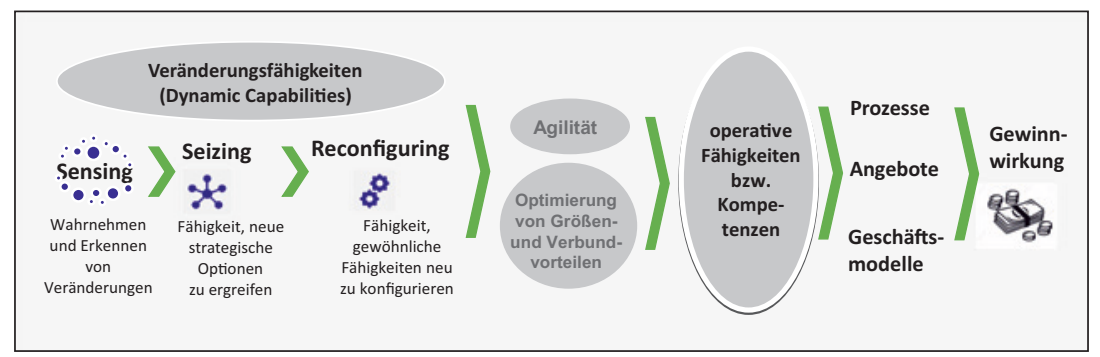

Abb. 6.21 Umsetzung digitaler Fähigkeiten durch (hybride) Agilität in der Organisation (Erweiterung von Abb. 4.1 und 4.3)

Die Fähigkeiten der organisationalen Agilität und der Optimierung von Größenund Verbundvorteilen können als Fähigkeit der „hybriden Agilität“ zusammengefasst werden (vgl. dazu auch Abschn. 4.1). ${ }^{26}$ Hybride Agilität ist eine Mischform zwischen 
- traditionellen linearen und sequenziellen Wasserfallansätzen, die alle Anforderungen (Umfang, Zeithorizont) an eine organisatorische Veränderung von Anfang an fixieren, und

- agilen iterativen Veränderungen über „Sprints“ zu minimal lebensfähigen Projekten (Abb. 6.22).

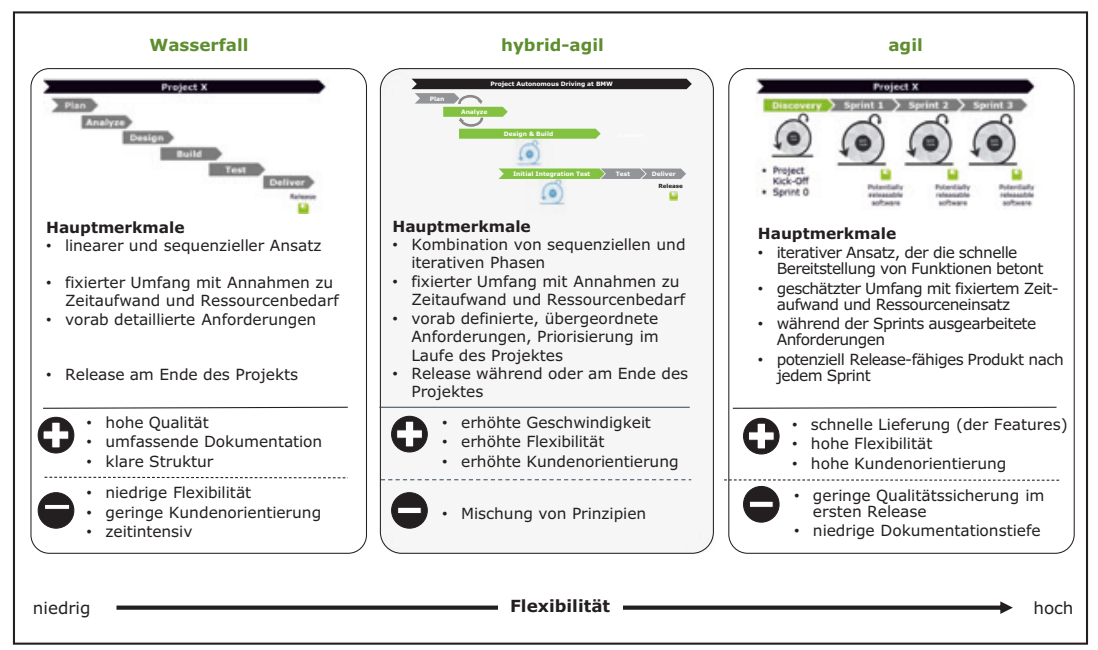

Abb. 6.22 Hybride Agilität. (Quelle: Proff u. a. 2019)

Hybride Agilität wird durch Veränderungsfähigkeiten ermöglicht und erlaubt Unternehmen in kapitalintensiven Branchen, die Handlungsspielräume der Digitalisierung durch digitale Transformation der Leistungen (Technologien und Prozesse, Angebote und Geschäftsmodelle) schneller zu nutzen ${ }^{27}$ (Abb. 6.22). In weniger kapitalintensiven Branchen, in denen die Optimierung von Größen- und Verbundvorteilen nicht so wichtig ist, reicht „einfache“ organisationale Agilität aus.

(Hybride) Agilität unterstützt damit sowohl die rasche Vernetzung und Skalierung von Leistungen über geeignete digitale Schlüssel als auch die schnelle Quantifizierung von anderen möglichen Einspar- und Erlöspotenzialen durch die Digitalisierung von Technologien und Prozessen, Angeboten und Geschäftsmodellen in Top-down-Ansätzen. Sie ist damit ein dritter Beschleuniger der digitalen Transformation. Auch dabei ist das Top-Management gefordert, nicht nur die großen Linien beim Sensing und Seizing zu definieren, sondern die Impulse durch Reconfiguring zu setzen und hybride Agilität zu treiben. 


\subsection{Accelerator of Digitalization (4): Schutz vor ungewolltem Datenabfluss (Cyber Security) erhöhen}

Ein vierter Ansatzpunkt zur Beschleunigung der digitalen Transformation ist die Erhöhung der Datensicherheit (Cyber Security). Denn die sinkenden Transaktionskosten durch die Digitalisierung erleichtern den in Kap. 2 unterstellten Datenaustausch bei der digitalen Transformation von Technologien und Prozessen, Angeboten und Geschäftsmodellen, gefährden damit jedoch die Datensicherheit.

Im Zentrum von Datenaustausch und Vernetzung liegt ein TechnologieStapel (Technology Stack) als Kern der Technologien, die zur Entwicklung und Implementierung von Lösungen in einem Unternehmen verwendet werden. ${ }^{28}$ Er ist durch eine komplizierte unternehmensspezifische Architektur mit verschiedenen Ebenen der Datenaufnahme und -verarbeitung gekennzeichnet. Diese Architektur kann von gemeinsamen IoT-Plattformen zur Datenspeicherung und Datennutzung über Data Analytics und künstliche Intelligenz bis hin zur Datenausgabe über Applikationen reichen (vgl. Abb. 6.23). Innerhalb dieser Architektur werden die Daten so verarbeitet, dass die Transaktionskosten der internen und externen Koordination möglichst gering sind (vgl. Kap. 2).

Dazu müssen die Daten in den Technologie-Stapeln schnell fließen, weil zu lange Reaktionszeiten zu einer Beeinträchtigung der Funktionsfähigkeit eines komplexen IoT-Systems führen können. Alleine für eine Simulation in der kleinen Deloitte Digital Factory mussten, wie oben erwähnt, Daten $40 \mathrm{Mal}$ um die Erde geschickt werden. Die dazu notwendige Zeit war teilweise zu lang, um integrierte Datenlösungen friktionslos zu ermöglichen. ${ }^{29}$ Deshalb gilt es, unterschiedliche Systeme friktionsfrei aufeinander abzustimmen, und zwar über

- ein MES verknüpfter IoT-Elemente wie z. B. kleiner, vernetzter Brillen, die am Körper getragen werden und den Arbeitsalltag des Trägers unterstützen sollen (Wearables),

- kollaborative Roboter (CoBots) und

- Technologien von Sender-Empfänger-Systemen wie Radio-FrequencyIdentification-(RFID-)Chips. 


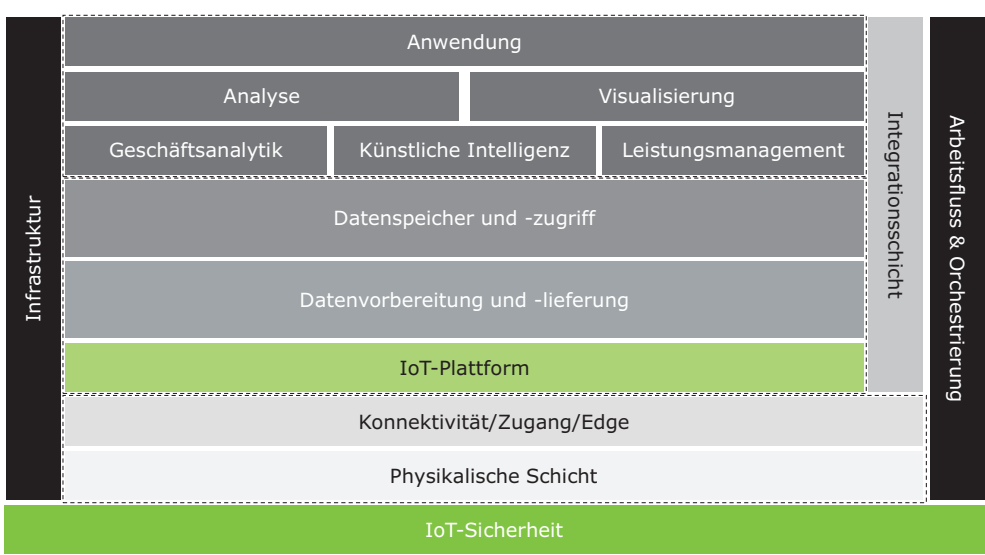

Abb. 6.23 Technologie-Stapel und Cyber Security. (Quelle: Deloitte Digital Factory)

Das ist bei weit entfernten Servern nicht immer ohne zeitliche Verzögerung möglich.

Wo derartig verknüpfte Technologie-Stapel vorhanden sind, bedarf es des Schutzes des internen Datenflusses, da z. B. ein Hackerangriff auf eine derart integrierte Datenarchitektur zu Produktionseinbrüchen, finanziellen Verlusten und Problemen bei Nichterfüllung regulatorischer Vorgaben führen würde. Die Folgen können für Unternehmen existenzbedrohend sein. In einer aktuellen globalen Umfrage $\mathrm{U}^{30}$

- gaben 83 Prozent der Unternehmen an, einen oder mehrere Angriffe pro Monat zu haben, fast 50 Prozent der Unternehmen erleben solche Angriffe täglich,

- mehr als 51 Prozent erlitten durch die Angriffe Produktionseinbußen,

- 2017 und 2018 entstanden Schäden von zusammen 43 Mrd. EUR und

- bei 40 Prozent der betroffenen Unternehmen entstand der größte Schaden durch einen Reputationsverlust.

Da man davon ausgehen kann, dass viele Unternehmen versuchen, Cyberangriffe geheim zu halten, sind diese Zahlen wohl nur die Spitze eines Eisberges - und schon sie lassen schwerwiegende Probleme und Gefahren erkennen. Diese liegen zunächst bei Hackerangriffen: Schafft es ein Hacker, in den Technologie-Stapel 
einzudringen und seine Schadsoftware dort zur Anwendung zu bringen, dann hat er z. B. Zugriff auf die Robotersteuerung in der über das MES vernetzten Produktion. Er kann einen Roboter so umprogrammieren, dass er z. B. ein fast fertiges Werkstück nicht an eine Fördereinheit übergibt, sondern es aus großer Höhe fallen lässt und damit einen Totalschaden verursacht. Gefahren liegen aber auch bereits in einem ungewollten Know-how-Abfluss durch undichte Stellen in der IT-Architektur.

Einen Schutz (Cyber Security) bieten z. B. Schutzwälle um die IT-Architektur sowie die Prozesse (vgl. dazu ebenfalls Abb. 6.23). Zunächst geht es um technische Lösungen wie sichere Datenzugänge, Firewalls, sichere Wartungsmethoden und die Durchführung von Penetrationstests. Daneben sind schnelle und sichere Notfallprozesse unabdingbar. Ebenfalls wichtig ist es, in der Cyber Security immer einen Schritt vor den von Hackern verwendeten Technologien zu sein, um keine größeren Lücken in der Abwehr aufreißen zu lassen. Schließlich müssen auch die Mitarbeiter trainiert werden, um Einfallstore zu verschließend und bei einem Sicherheitsvorfall möglichst schnell Gegenmaßnahmen einleiten zu können. ${ }^{31}$ Richtlinien und Notfallpläne müssen aufgestellt und in den Unternehmen eingeübt werden. Damit unterscheidet sich die Cyber Security nicht von anderen Ansätzen des Krisenmanagements. ${ }^{32}$ Zusätzlich ist es notwendig, die Cyber-Kompetenz der Unternehmensführung und des Aufsichtsrats zu stärken. ${ }^{33}$ Dazu ist aber ein Mindest-Know-how erforderlich.

Gelingt die Verstärkung der Cyber Security nicht, wird auch die digitale Transformation nicht zügig vorankommen. Umgekehrt kann eine gute Cyber Security als vierter Ansatzpunkt einer beschleunigten digitalen Transformation gesehen werden. Cyber Security ist ebenfalls Aufgabe des Top-Managements, v. a. die Allokation von Investitionsmitteln. Viele Unternehmen warten allerdings, bis sie erstmals von einem Hackerangriff schwer geschädigt werden, bevor sie sich der Cyber Security annehmen und damit die digitale Transformation beschleunigen.

Alle vier Ansatzpunkte zur Beschleunigung der Digitalisierung (Abschn. 6.3 bis 6.6) sind unabhängig von der digitalen Reife bzw. von der Zugehörigkeit zu einem der Archetypen digitaler Unternehmen (Abschn. 5.2) und von möglichen Wegen der digitalen Transformation (Abschn. 6.1). Sie helfen den Unternehmen aber dabei, diese Wege möglichst schnell zu beschreiten und damit verstärkte Wettbewerberreaktionen (Abschn. 6.2) zu vermeiden - zumindest, wenn das TopManagement dahintersteht. 


\section{Anmerkungen zu Kapitel 6}

1. Vgl. Knobbe, Proff (2020).

2. Vgl. zu Entscheidungen unter Unsicherheit z. B. Proff u. a. (2014, S. 12-16).

3. Vgl. Brezis u. a. (1991).

4. Vgl. dazu Proff (2007, S. 20) oder auch Bogner u. a. (1996).

5. Vgl. Rogers (2016).

6. Vgl. www.wissenschaftsforum.uni-due-de

7. Vgl. dazu Thonemann (2015).

8. Die Effekte 1. bis 3. sind nicht additiv zu sehen.

9. Vgl. z. B. Zahra u. a. (2006: S. 919) und Teece u. a. (2016).

10. Vgl. z. B. Overby u. a. (2006) und Chakravarty u. a. (2013).

11. Vgl. Teece u. a. (2016).

12. Vgl. ebd. (S. 25).

13. Vgl. ebd. (S. 21).

14. Vgl. auch hierzu Teece u. a. (2016).

15. Vgl. Malik (2015).

16. Vgl. Leiblein, Miller (2003), Afuah (2001) und Kachaner, Whybrew (2014).

17. Vgl. Tücks (2010), Burggräf u. a. (2016) oder Becker u. a. (2016).

18. Vgl. z. B. Dixit (1979).

19. Vgl. z. B. Knips (1970).

20. Vgl. Teece u. a. (2016, S. 41 und S. 29).

21. Vgl. ebd.

22. Vgl. ebd.

23. Vgl. Knobbe, Proff (2020).

24. Vgl. Carillo, Franza (2006) oder Becker u. a. (2016).

25. Vgl. z. B. Leiblein, Miller (2003), Afuah (2001) oder Burggräf u. a. (2016).

26. Vgl. Knobbe, Proff (2020).

27. Vgl. Teece u. a. (2016), Lessard u. a. (2016).

28. Vgl. zur Cybersecurity Bartsch, Frey (2018).

29. Vgl. dazu Abb. 6-18 zu den (IT)Problemen der digitalen Transformation in Abschn. 6.3.

30. Vgl. die Cyber Sicherheitsumfrage (Bitkom e. V. 2019).

31. Vgl. Deloitte (2017).

32. Vgl. Proff, Proff (2013).

33. Vgl. Deloitte (2017). 
Open Access Dieses Kapitel wird unter der Creative Commons Namensnennung 4.0 International Lizenz (http://creativecommons.org/licenses/by/4.0/deed.de) veröffentlicht, welche die Nutzung, Vervielfältigung, Bearbeitung, Verbreitung und Wiedergabe in jeglichem Medium und Format erlaubt, sofern Sie den/die ursprünglichen Autor(en) und die Quelle ordnungsgemäß nennen, einen Link zur Creative Commons Lizenz beifügen und angeben, ob Änderungen vorgenommen wurden.

Die in diesem Kapitel enthaltenen Bilder und sonstiges Drittmaterial unterliegen ebenfalls der genannten Creative Commons Lizenz, sofern sich aus der Abbildungslegende nichts anderes ergibt. Sofern das betreffende Material nicht unter der genannten Creative Commons Lizenz steht und die betreffende Handlung nicht nach gesetzlichen Vorschriften erlaubt ist, ist für die oben aufgeführten Weiterverwendungen des Materials die Einwilligung des jeweiligen Rechteinhabers einzuholen.

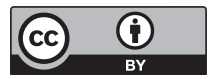

